\title{
Effect of Aspect Ratio and Boundary Conditions in Modeling Shape Memory Alloy Nanostructures with 3D Coupled Dynamic Phase-Field Theories
}

\author{
R. Dhote, ${ }^{1}$ H. Gomez, ${ }^{2}$ R. Melnik, ${ }^{3}$ and J. Zu ${ }^{1}$ \\ ${ }^{1}$ Mechanical and Industrial Engineering, University of Toronto, 5 King's College Road, Toronto, ON, Canada M5S 3G8 \\ ${ }^{2}$ Department of Applied Mathematics, University of A Coruña, Campus de Elvina, s/n, 15192 A Coruña, Spain \\ ${ }^{3}$ MS2Discovery Interdisciplinary Research Institute, M2NeT Laboratory, Wilfrid Laurier University, Waterloo, ON, Canada N2L 3C5
}

Correspondence should be addressed to H. Gomez; hgomez@udc.es

Received 20 June 2016; Revised 10 September 2016; Accepted 19 September 2016

Academic Editor: Mohsen Asle Zaeem

Copyright (C) 2016 R. Dhote et al. This is an open access article distributed under the Creative Commons Attribution License, which permits unrestricted use, distribution, and reproduction in any medium, provided the original work is properly cited.

\begin{abstract}
The behavior of shape memory alloy (SMA) nanostructures is influenced by strain rate and temperature evolution during dynamic loading. The coupling between temperature, strain, and strain rate is essential to capture inherent thermomechanical behavior in SMAs. In this paper, we propose a new 3D phase-field model that accounts for two-way coupling between mechanical and thermal physics. We use the strain-based Ginzburg-Landau potential for cubic-to-tetragonal phase transformations. The variational formulation of the developed model is implemented in the isogeometric analysis framework to overcome numerical challenges. We have observed a complete disappearance of the out-of-plane martensitic variant in a very high aspect ratio SMA domain as well as the presence of three variants in equal portions in a low aspect ratio SMA domain. The dependence of different boundary conditions on the microstructure morphology has been examined energetically. The tensile tests on rectangular prism nanowires, using the displacement based loading, demonstrate the shape memory effect and pseudoelastic behavior. We have also observed that higher strain rates, as well as the lower aspect ratio domains, resulting in high yield stress and phase transformations occur at higher stress during dynamic axial loading.
\end{abstract}

\section{Introduction}

Shape memory alloys (SMAs) are metallic alloys with distinguished characteristics like thermoelastic phase transformations, unique shape memory effect and pseudoelastic hysteretic behaviors, excellent corrosion resistance, biocompatibility along with high strength, strain, and power density. SMAs have been widely used as transducers in sensing and actuation applications $[1,2]$. More recently, they found applications in SMA nanotubes [3], nanofilms [4-6], and nanowires [7-9] for nanoelectromechanical and microelectromechanical systems and biomedical devices as well as for a number of other applications [10-13]. In many of the above applications, SMAs are actuated dynamically at different loading rates under various boundary conditions. Factors, like geometry [14], boundary conditions $[5,9,15]$, and loading rates [16], have substantial influence on the microstructure evolution and their thermomechanical behaviors. However, these factors have not been studied in a fully coupled thermomechanical framework for SMAs. Such studies would aid the understanding of phase transformation mechanisms and thermomechanical behavior of SMAs for better SMAbased application developments.

SMAs have inherent thermomechanical properties. In the various experimental studies, the sensitivity of dynamic loading and strong interactions of the thermal and mechanical effects have been emphasized for SMA specimens [16-23]. The drastic changes in a fundamental mechanical response of SMAs have been attributed to self-heating and cooling due to exothermic and endothermic processes during latent heat transfer. However, the experimental setups for dynamic loading behavior are complex and time consuming. Arguably, the modeling framework is a complementary tool to study the 
dynamic behavior, its influence on microstructure evolution, and thermomechanical response of SMAs.

Several modeling approaches have significantly contributed to the understanding of temperature- and stressinduced transformations [1, 2, 24-26]. Extensive discussions of various models have been reviewed in $[27,28]$. Here we are interested in phase-field (PF) models, which are used across different length scales, including the nanoscale [29-31]. The PF models provide a unified framework that describes stressand temperature-induced phase transformations, including their dynamics. The characteristic features of transformations have been thoroughly studied, in particular, in the context of nucleation, growth, domain wall movement, merging, and elimination $[32,33]$. In the literature, there have been extensive studies of 2D SMA PF models under quasistatic loading conditions. The 2D models can qualitatively capture the basic mechanisms during phase transformations, under the assumption of constant strain across the out-of-plane direction. However, the real-life applications often require $3 \mathrm{D}$ geometries, where the strain can be accommodated in all three directions. At the same time, very few 3D studies have been reported in the literature for quasistatic and dynamic loading conditions.

An important pioneering work on the 3D PF model was reported by Barsch and Krumhansl $[34,35]$. They introduced the strain-based order parameters (OPs) model to describe a cubic-to-tetragonal phase transformation consisting of inhomogeneous strain field associated with domain walls, constituent phases, and transformation precursors. Later, Jacobs et al. [36] incorporated the Rayleigh dissipation and solved the isothermal model to obtain static domain patterns during temperature induced transformations. The computer simulations on a large domain revealed several microstructure features observed experimentally. Wang and Khachaturyan [37] described a 3D PF model that accounts for the transformation induced elastic strain of martensitic phase transformations. They developed a stochastic field kinetic model and studied the nucleation growth of martensitic evolution in cubic-to-tetragonal transformations. Later, Artemev et al. [38] investigated the effect of external load on phase transformations in a polycrystalline material.

Typically, the strain-based order parameter model describes pseudoplastic regime qualitatively [26], but unloading of reverse PT from martensite to austenite is not described. Both phases lose their stability at zero elastic moduli. In addition, the transformation strain tensor using the Landau potential is required to be stress and temperature independent as per crystallographic theory of martensitic PT. The transformation strain at phase equilibrium in the Landau theory is not prescribed independently but depends on equilibrium values of the OP that are obtained from the thermodynamic potentials. These important considerations are addressed by Levitas et al. in their model [30, 39, 40]. They developed an advanced thermodynamic potential based on the transformation strain-related order parameters that capture experimentally observed stress-strain curves, all the temperature dependent thermomechanical properties of austenite and martensitic variants, and phase transformation between austenite and martensitic variants, as well as between martensitic variants with arbitrary types of symmetry. Idesman et al. [41] used the model and demonstrated the effect of inertial forces that can alter the microstructure during evolution. Later, multiphase PF theories for temperature- and stress-induced PTs and the surface tension at interfaces are described in $[42,43]$.

Following the strain-based order parameter modeling frameworks [34, 36], Ahluwalia et al. [44, 45] reported the only study at that time showing dynamic strain loading characteristics of SMAs. However, the influence of different strain rates on microstructure and mechanical response of FePd samples on temperature- and stress-induced PTs was examined by using exclusively the isothermal model. Other 3D PF models have reported morphological evolution in spinodal decomposition [46], thermoelastic transformations [47], and decomposition of the supersaturated binary solid solution [48].

Most of the above 3D models examined the phase transformations under static or quasistatic conditions in a controlled temperature environment with periodic boundary conditions on simple cubic geometries. With the inherent thermomechanical coupling and other factors mentioned above, it is essential to model the thermomechanical physics and the rate dependent coupling in a single framework. Recently, for the first time, we presented a coupled thermomechanical framework for modeling 2D square-torectangular phase transformations [49] and developed a new computational framework, based on the isogeometric analysis (using nonuniform rational B-spline (NURBS) basis functions) [50]. In this work, we extend this new framework to the 3D model for cubic-to-tetragonal phase transformations and numerically solve the resulting coupled, strongly nonlinear equations by using the isogeometric analysis (IGA). A point to remark is that the energy functionals used in the $2 \mathrm{D}$ [50] and 3D models must be distinct in order to satisfy the symmetry related to martensitic variants in the energy landscape. This leads to distinct expressions for constitutive relationships and the thermomechanical coupling term. The 3D constitutive equations and thermomechanical coupling term are not reducible to the $2 \mathrm{D}$ constitutive equations and the thermomechanical coupling term due to the selection of distinct functionals. Consequently, this leads to different models and distinct numerical implementations for the $2 \mathrm{D}$ and 3D models.

In this paper, we first provide the derivation of the 3D fully coupled thermomechanical model for the cubicto-tetragonal martensitic transformations in Section 2. The numerical formulation of the developed model using isogeometric analysis is presented in Section 3. The effect of aspect ratio and boundary conditions on microstructure morphology and energetics is discussed during the temperature induced phase transformations in Section 4. Section 5 describes the effect of aspect ratio and strain rate sensitivity on thermomechanical behavior of SMAs during stress-induced transformations. Finally, the conclusions are summarized in Section 6. 


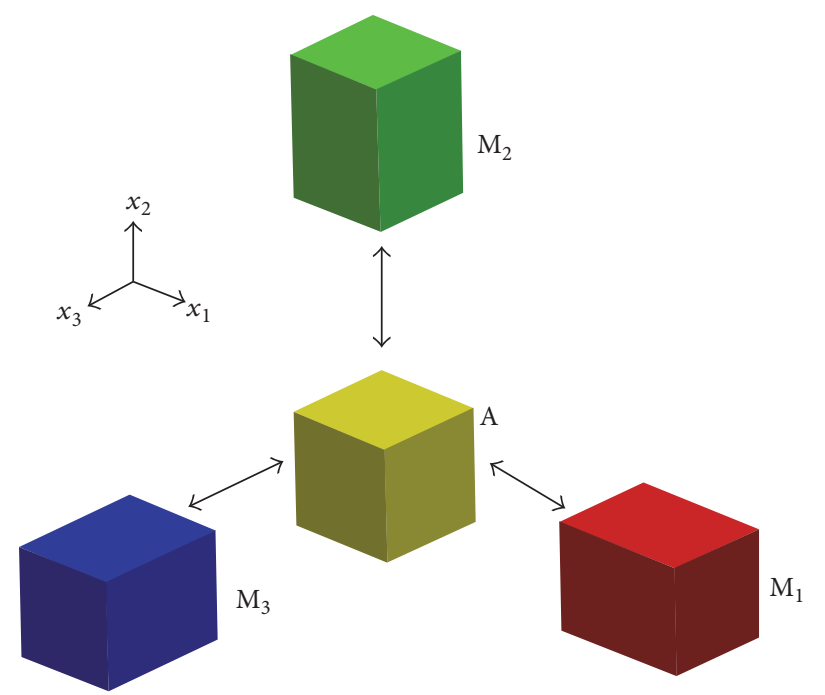

(a)

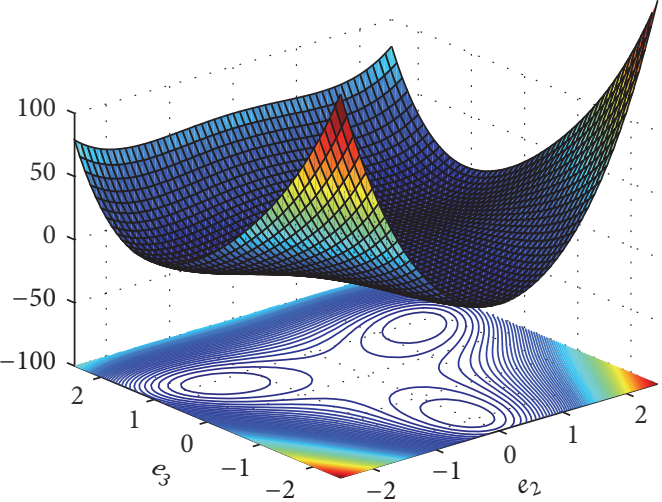

(b)

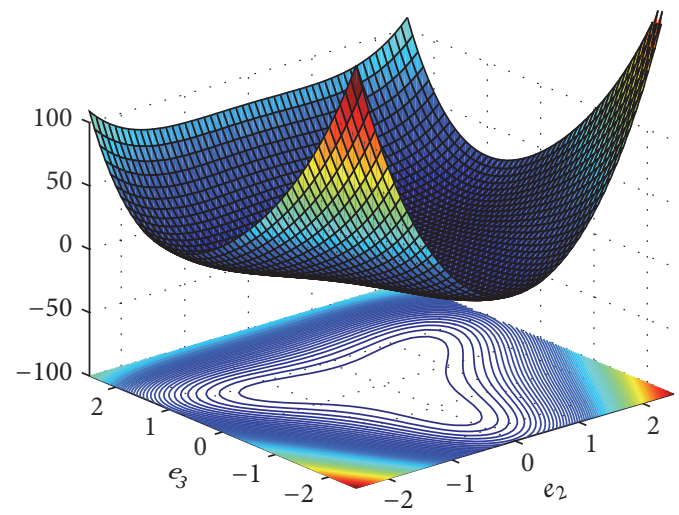

(c)

FiguRE 1: Cubic-to-tetragonal phase transformations (a) schematic of microstructures (austenite (A), martensite variants $\left(M_{1}, M_{2}, M_{3}\right)$ ), and $\mathscr{F}_{L}$ plot at (b) $\tau=-1.2$ and (c) $\tau=1.2$.

\section{3D Thermomechanical Phase-Field Model}

The thermomechanical properties of SMAs are functions of their microstructures and loadings. The properties can be described by a phase-field model using the Ginzburg-Landau approach [28]. As different parts of the domain may evolve into distinct phases, a continuum description of phases in the model can be carried out by introducing a diffuse interface domain wall between phases as a function of $\mathrm{OP}(\mathrm{s})$. By choosing appropriate OPs, all possible phases in a domain can be described. Unlike other approaches [30, 37], here we describe the cubic-to-tetragonal phase transformations in a PF model with respect to the strain-based (deviatoric) OPs. The cubic-to-tetragonal phase transformation can be schematically depicted as shown in Figure 1(a), with the cubic austenite $(\mathrm{A})$ phase and the three tetragonal martensite variants $\left(M_{1}, M_{2}\right.$, and $\left.M_{3}\right)$ stretched along the three rectilinear directions. The thermomechanical behavior of SMAs is established by solving a coupled 3D model described below.

We begin by introducing basic notations for the kinematics of SMAs. Let us declare $\mathbf{u}=\left\{u_{1}, u_{2}, u_{3}\right\}^{T}$ the displacement field. We will work on the physical domain $\Omega \subset \mathbb{R}^{3}$, which is assumed to be an open set parameterized by Cartesian coordinates $\mathbf{x}=\left\{x_{1}, x_{2}, x_{3}\right\}^{T}$. We define the strain measures $e_{i}$, for $i=1, \ldots, 6$, using the symmetric strain tensor as follows:

$$
\left\{\begin{array}{l}
e_{1} \\
e_{2} \\
e_{3} \\
e_{4} \\
e_{5} \\
e_{6}
\end{array}\right\}=\left[\begin{array}{l|l}
\mathbb{D}_{3} & \mathbb{O}_{3} \\
\hline \mathbb{O}_{3} & \mathbb{D}_{3}
\end{array}\right]\left\{\begin{array}{l}
\epsilon_{11} \\
\epsilon_{22} \\
\epsilon_{33} \\
\epsilon_{23} \\
\epsilon_{13} \\
\epsilon_{12}
\end{array}\right\},
$$

where $\mathbb{D}_{3}, \mathbb{O}_{3}, \mathbb{D}_{3}$ are the $3 \times 3$ constant matrices. In particular,

$$
\mathbb{D}_{3}=\left[\begin{array}{ccc}
\frac{1}{\sqrt{3}} & \frac{1}{\sqrt{3}} & \frac{1}{\sqrt{3}} \\
\frac{1}{\sqrt{2}} & -\frac{1}{\sqrt{2}} & 0 \\
\frac{1}{\sqrt{6}} & -\frac{1}{\sqrt{6}} & -\frac{2}{\sqrt{6}}
\end{array}\right],
$$




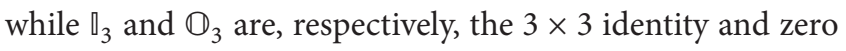
matrices.

We call $e_{1}$ hydrostatic strain, $e_{2}, e_{3}$ deviatoric strains, and $e_{4}, e_{5}, e_{6}$ shear strains. In (1), $\epsilon$ denotes the Cauchy-Lagrange infinitesimal strain tensor, whose components are $\epsilon_{i j}=\left(u_{i, j}+\right.$ $\left.u_{j, i}\right) / 2, i, j \in\{1,2,3\}$, where an inferior comma denotes partial differentiation (e.g., $u_{i, j}=\partial u_{i} / \partial x_{j}$ ). The deviatoric strains $e_{2}$ and $e_{3}$ are selected as the OPs to describe different phases in a domain.

We use the free-energy functional initially proposed by Barsch and Krumhansl [34] and later modified by Ahluwalia et al. [45] to study the cubic-to-tetragonal martensitic transformations in SMAs. The free energy functional $\mathscr{F}$ for the cubic-to-tetragonal transformations is written as

$$
\mathscr{F}[\mathbf{u}, \theta]=\int_{\Omega}\left[\mathscr{F}_{b}+\mathscr{F}_{s}+\mathscr{F}_{L}+\mathscr{F}_{g}\right] \mathrm{d} \Omega,
$$

where the bulk $\mathscr{F}_{b}$, the shear $\mathscr{F}_{s}$, the Landau $\mathscr{F}_{L}$, and the Ginzburg $\mathscr{F}_{g}$ energy contributions are defined as

$$
\begin{aligned}
& \mathscr{F}_{b}=\frac{a_{1}}{2} e_{1}^{2}, \\
& \mathscr{F}_{s}=\frac{a_{2}}{2}\left(e_{4}^{2}+e_{5}^{2}+e_{6}^{2}\right), \\
& \mathscr{F}_{L}=a_{3} \tau\left(e_{2}^{2}+e_{3}^{2}\right)+a_{4} e_{3}\left(e_{3}^{2}-3 e_{2}^{2}\right)+a_{5}\left(e_{2}^{2}+e_{3}^{2}\right)^{2}, \\
& \mathscr{F}_{g}=\frac{k_{g}}{2}\left(\left|\nabla e_{2}\right|^{2}+\left|\nabla e_{3}\right|^{2}\right) .
\end{aligned}
$$

Here, $a_{i}, i \in\{1, \ldots, 5\}$ and $k_{g}$ are material parameters, $\tau$ is the dimensionless temperature defined as $\tau=(\theta-$ $\left.\theta_{m}\right) /\left(\theta_{0}-\theta_{m}\right)$, where $\theta_{0}$ and $\theta_{m}$ are the material properties specifying the transformation start and end temperatures, and $|\cdot|$ denotes the Euclidean norm of a vector. The $\mathscr{F}_{b}$ and $\mathscr{F}_{s}$ energy components represent non-OP contributions due to the bulk and shear energies, respectively. The $\mathscr{F}_{L}$ corresponds to the OP contribution and represents the nonconvex 2-34 polynomial Landau functional. The Landau functional is dependent on the temperature coefficient $\tau$. The $\mathscr{F}_{L}$ is plotted for two different $\tau$ values corresponding to the martensite and the austenite phases in Figures 1(b), and 1(c), respectively.

The kinetic energy $\mathscr{K}$, the energy associated with the external body forces $\mathscr{B}$, and the Raleigh dissipation $\mathscr{R}$ are defined, respectively, as

$$
\begin{aligned}
& \mathscr{K}[\dot{\mathbf{u}}]=\int_{\Omega} \frac{\rho}{2}|\dot{\mathbf{u}}|^{2} \mathrm{~d} \Omega, \\
& \mathscr{B}[\mathbf{u}]=-\int_{\Omega} \mathbf{f} \cdot \mathbf{u} \mathrm{d} \Omega, \\
& \mathscr{R}[\dot{\mathbf{u}}]=\int_{\Omega} \frac{\eta}{2}|\dot{\mathbf{e}}|^{2} \mathrm{~d} \Omega,
\end{aligned}
$$

where a dot over a function denotes partial differentiation with respect to time, $\rho$ is the density, $\mathbf{f}$ is the body load vector, $\eta$ is the dissipation coefficient, and $\mathbf{e}=\left\{e_{i}\right\}_{i=1, \ldots, 6}$. The potential energy of the system $\mathscr{U}$ is defined as $\mathscr{U}[\mathbf{u}]=\mathscr{F}[\mathbf{u}]+$ $\mathscr{B}[\mathbf{u}]$, while the Lagrangian takes on the form $\mathscr{L}[\mathbf{u}, \dot{\mathbf{u}}]=$
$\mathscr{K}[\dot{\mathbf{u}}]-\mathscr{U}[\mathbf{u}]$. Let us define the Hamiltonian of the system $\mathscr{H}$ as

$$
\mathscr{H}[\mathbf{u}, \dot{\mathbf{u}}]=\int_{0}^{t} \mathscr{L}[\mathbf{u}, \dot{\mathbf{u}}] \mathrm{d} t,
$$

where $[0, t]$ is the time interval of interest. Following a variational approach, the governing equation of motion has the form

$$
\frac{\partial}{\partial t}\left(\frac{\delta \mathscr{L}}{\delta \dot{\mathbf{u}}}\right)-\frac{\delta \mathscr{L}}{\delta \mathbf{u}}=-\frac{\delta \mathscr{R}}{\delta \dot{\mathbf{u}}},
$$

where the operators $\delta(\cdot) / \delta \mathbf{u}$ and $\delta(\cdot) / \delta \dot{\mathbf{u}}$ denote the variational derivatives with respect to $\mathbf{u}$ and $\dot{\mathbf{u}}$, respectively. Note that setting the right-hand side of (7) to zero yields the Lagrange general equation of motion. However, we let the system relax according to the Lagrange equation of motion using the Raleigh dissipation $\mathscr{R}$.

After analytic manipulations, (7) may be written as

$$
\rho \frac{\partial^{2} \mathbf{u}}{\partial t^{2}}=\nabla \cdot \boldsymbol{\sigma}+\eta \nabla \cdot \boldsymbol{\sigma}^{\prime}+\nabla \cdot \boldsymbol{\sigma}_{w}+\mathbf{f} .
$$

The symmetric stress tensor $\sigma=\left\{\sigma_{i j}\right\}$ components are defined in Appendix 6, specifically for the cubic-to-tetragonal PTs. The equations for $\sigma_{i j}$ in terms of the strains are strongly nonlinear. The cubic nonlinearity allows us to capture the hysteretic phase transformation properties like shape memory effect and pseudoelasticity as a function of temperature. The stress expressions define material constitutive laws of the phase transformation.

The symmetric dissipation stress tensor $\boldsymbol{\sigma}^{\prime}=\left\{\sigma_{i j}^{\prime}\right\}$ is a linear function of the strain rates $\dot{e}_{i}, i=1, \ldots, 6$ defined as

$$
\left\{\begin{array}{c}
\sigma_{11}^{\prime} \\
\sigma_{22}^{\prime} \\
\sigma_{33}^{\prime} \\
\sigma_{23}^{\prime} \\
\sigma_{13}^{\prime} \\
\sigma_{12}^{\prime}
\end{array}\right\}=\left[\begin{array}{l|l|l}
\mathbb{D}_{3}^{T} & \mathbb{O}_{3} \\
\hline \mathbb{O}_{3} & \frac{1}{2} \mathbb{D}_{3}
\end{array}\right]\left\{\begin{array}{c}
\dot{e}_{1} \\
\dot{e}_{2} \\
\dot{e}_{3} \\
\dot{e}_{4} \\
\dot{e}_{5} \\
\dot{e}_{6}
\end{array}\right\} .
$$

The fourth-order differential terms in (8) coming from $\nabla$. $\sigma_{w}$ represent domain walls between different phases in a domain. The additional stress components corresponding to the gradient terms in the potential energy take on the form

$$
\boldsymbol{\sigma}_{w}=\frac{k_{g}}{3} \nabla^{2}\left(\nabla^{T} \mathbf{u}-3 \nabla_{d} \mathbf{u}\right),
$$

where $\nabla^{\mathrm{T}} \mathbf{u}$ denotes the transpose of the displacement gradient (i.e., $\nabla^{T} \mathbf{u}=\left\{u_{j, i}\right\}$ ). We also use the notation $\nabla_{d} \mathbf{u}=$ $\operatorname{diag}\left(u_{1,1}, u_{2,2}, u_{3,3}\right)$, where $\operatorname{diag}(a, b, c)$ is a $3 \times 3$ diagonal matrix whose diagonal entries starting in the upper left corner are $a, b, c$.

The governing equation of the thermal field is obtained from the conservation law for internal energy $\iota$ [51] as

$$
\rho \frac{\partial \iota}{\partial t}-\boldsymbol{\sigma}^{T}: \nabla \dot{\mathbf{u}}+\nabla \cdot \mathbf{q}=g,
$$


where $\mathbf{q}=-\kappa \nabla \theta$ is the Fourier heat flux vector, $\kappa$ is the heat conductance coefficient of the material, and $g$ is a thermal loading. The internal energy is connected with the potential energy constructed above via the Helmholtz free energy $\Psi$ as

$$
\begin{aligned}
\iota & =\Psi(\theta, \epsilon)-\theta \frac{\partial \Psi(\theta, \epsilon)}{\partial \theta}, \\
\Psi(\theta, \epsilon) & =\mathscr{L}[\mathbf{u}, \dot{\mathbf{u}}]-C_{v} \theta \ln (\theta),
\end{aligned}
$$

where $C_{v}$ is the specific heat of the material. On substituting the above relationships in (11), the governing equation of the thermal field is formulated as

$$
C_{v} \frac{\partial \theta}{\partial t}=\kappa \nabla^{2} \theta+\Xi \theta\left(\nabla \cdot \mathbf{u} \nabla \cdot \dot{\mathbf{u}}-3 \operatorname{tr}\left(\nabla_{d} \mathbf{u} \nabla_{d} \dot{\mathbf{u}}\right)\right)+g
$$

where the operator $\operatorname{tr}(\cdot)$ denotes the trace of a square matrix, and we have assumed $\kappa$ to be constant. The second term on the right-hand side of (13) is a nonlinear term, which couples temperature, deformation gradient (strain), and rate of the deformation gradient (strain rate). Hence, (8) and (13) describe the thermomechanical physics of SMAs by creating the two-way coupling via $\theta, \nabla \mathbf{u}$ and $\nabla \dot{\mathbf{u}}$.

In what follows, we use the component-wise version of the governing equations (8) and (13). Let us define the tensor $\boldsymbol{\mu}=$ $\left\{\mu_{i j}\right\}$, such that $\boldsymbol{\mu}=\left(k_{g} / 3\right)\left(\nabla^{T} \mathbf{u}-3 \nabla_{d} \mathbf{u}\right)$, and $\boldsymbol{\sigma}_{w}=\nabla^{2} \boldsymbol{\mu}$. Thus, the governing equations may be written in the component form as

$$
\begin{aligned}
\rho \ddot{u}_{i} & =\sigma_{i j, j}+\eta \sigma_{i j, j}^{\prime}+\mu_{i j, k k j}+f_{i}, \\
C_{v} \dot{\theta} & =\kappa \theta_{, i i}+\Xi \theta\left(u_{i, i} \dot{u}_{j, j}-3 u_{i, i} \dot{u}_{i, i}\right)+g,
\end{aligned}
$$

where repeated indices indicate summation. The governing thermomechanical equations (14a) and (14b) are converted into the dimensionless form as

$$
\begin{gathered}
\rho \ddot{\bar{u}}_{i}=\bar{\sigma}_{i j, j}+\bar{\eta} \bar{\sigma}_{i j, j}^{\prime}+\bar{\mu}_{i j, k k j}+\bar{f}_{i}, \\
\bar{C}_{v} \dot{\bar{\theta}}=\bar{\kappa} \bar{\theta}_{, i i}+\bar{\Xi} \bar{\theta}\left(\bar{u}_{i, i} \dot{\bar{u}}_{j, j}-3 \bar{u}_{i, i} \dot{\bar{u}}_{i, i}\right)+\bar{g}
\end{gathered}
$$

by using the following change of variables:

$$
\begin{aligned}
e_{i} & =e_{c} \overline{e_{i}}, \\
u_{i} & =e_{c} \delta \overline{u_{i}}, \\
x & =\delta \bar{x}, \\
\mathscr{F} & =\mathscr{F}_{c} \overline{\mathscr{F}}, \\
t & =t_{c} \bar{t}, \\
\theta & =\theta_{c} \bar{\theta},
\end{aligned}
$$

with the scaling constants defined as

$$
\begin{aligned}
\delta & =\sqrt{\frac{k_{g}}{a_{0}}}, \\
\bar{a}_{1} & =\frac{a_{1}}{a_{0}}, \\
\bar{a}_{2} & =\frac{a_{2}}{a_{0}}, \\
\bar{a}_{4} & =2, \\
\bar{a}_{5} & =1, \\
\mathscr{F}_{c} & =\delta^{2} e_{c}^{2} a_{0}, \\
\bar{\eta} & =\frac{\eta}{a_{0}} \sqrt{\frac{a_{0}}{\rho \delta^{2}}}, \\
\overline{C_{v}} & =\frac{\rho C_{v} \tau}{t_{c}}, \\
\bar{\kappa} & =\frac{\kappa \tau}{\delta^{2} \overline{C_{v}}}, \\
\bar{\Xi} & =-\frac{2}{3} \frac{a_{0} e_{c}^{2}}{t_{c} \overline{C_{v}}} .
\end{aligned}
$$

The rescaled free energy takes the form

$$
\begin{aligned}
\overline{\mathscr{F}}= & \frac{\bar{a}_{1}}{2}\left(\bar{e}_{1}\right)^{2}+\frac{\bar{a}_{2}}{2}\left(\bar{e}_{4}^{2}+\bar{e}_{5}^{2}+\bar{e}_{6}^{2}\right)+\bar{a}_{3} \tau\left(\bar{e}_{2}^{2}+\bar{e}_{3}^{2}\right) \\
& +\bar{a}_{4} \bar{e}_{3}\left(\bar{e}_{3}^{2}-3 \bar{e}_{2}^{2}\right)+\bar{a}_{5}\left(\bar{e}_{2}^{2}+\bar{e}_{3}^{2}\right)^{2} \\
& +\frac{\bar{k}_{g}}{2}\left[\left|\nabla \bar{e}_{2}\right|^{2}+\left|\nabla \bar{e}_{3}\right|^{2}\right] .
\end{aligned}
$$

In the rest of the paper, we drop the bar symbol over the dimensionless variables for simplicity.

\section{Numerical Formulation}

The governing equations (15a) and (15b) are highly nonlinear to account for the hysteretic behavior with thermomechanical coupling and fourth-order differential terms in the general 3D formulation. These complexities present a number of numerical challenges. We have developed an IGA framework that allows the straightforward solution to the fourth-order equations. It also allows the use of coarser meshes, larger time steps along with geometrical flexibility and accuracy [52]. The IGA numerical implementation of (15a) and (15b) are described in detail in [53].

Our computational method for the structural equations is based on the following principle of virtual work

$$
\delta W\left(\delta u_{i}, u_{i}\right)=\delta W^{\mathrm{int}}-\delta W^{\mathrm{ext}}+\delta W^{\mathrm{kin}}=0, \quad \forall \delta u_{i}
$$


where the $\delta u_{i}$ 's are the virtual displacements and

$$
\begin{aligned}
& \delta W^{\text {int }}=\int_{\Omega} \delta \epsilon_{i j}\left(\sigma_{i j}+\eta \sigma_{i j}^{\prime}\right) \mathrm{d} \Omega-\int_{\Omega} \delta u_{i, j k} \mu_{i j, k} \mathrm{~d} \Omega, \\
& \delta W^{\text {ext }}=\int_{\Omega} \delta u_{i} f_{i} \mathrm{~d} \Omega, \\
& \delta W^{\text {kin }}=\int_{\Omega} \delta u_{i} \rho \ddot{u}_{i} \mathrm{~d} \Omega .
\end{aligned}
$$

Here $\delta \epsilon$ denotes the strain tensor associated with the virtual displacements $\delta \mathbf{u}$.

For the thermal physics, we use the variational principle

$$
\begin{gathered}
\int_{\Omega} \delta \theta\left(c_{v} \dot{\theta}-\Xi \theta\left(u_{i, i} \dot{u}_{j, j}-3 u_{i, i} \dot{u}_{i, i}\right)\right) \mathrm{d} \Omega \\
+\int_{\Omega} \delta \theta_{, i} \kappa \theta_{, i} \mathrm{~d} \Omega=0, \quad \forall \delta \theta .
\end{gathered}
$$

Note that, for the integrals in $\delta W^{\text {int }}$ to be well defined, the strains must be globally smooth, which cannot be easily achieved using the classical finite element method. To overcome this difficulty, we propose a numerical method based on the isogeometric analysis, a methodology in computational mechanics that permits globally continuous representations of strains.

To completely define our algorithm, we need to specify discrete versions of the displacements and temperature. We use the following expressions:

$$
\begin{aligned}
& u_{i}^{h}(\mathbf{x}, t)=\sum_{A=1}^{n_{b}} u_{i}^{A}(t) N^{A}(\mathbf{x}) ; \\
& \theta^{h}(\mathbf{x}, t)=\sum_{A=1}^{n_{b}} \theta^{A}(t) N^{A}(\mathbf{x}),
\end{aligned}
$$

where the superscript $h$ indicates a discrete field and $n_{b}$ is the dimension of the discrete space. We note that identical approximations are used for the virtual counterparts of $u_{i}^{h}$ and $\theta^{h}$. The $N^{A}$ 's are B-spline (or NURBS) basis functions. Using the rich basis functions, the IGA allows a straightforward implementation of the fourth order equations.

We illustrated the details about the IGA numerical implementation of the developed model, the geometrical flexibility, accuracy, and robustness of the new numerical formulation in [53]. In this paper, we focus on thermomechanical behavior of rectangular prism SMA nanostructures subjected to temperature- and stress-induced loadings, with our special attention to the effects of aspect ratio and boundary conditions. The nomenclature of domains and boundaries is presented in Figure 2. The simulations have been conducted on $\mathrm{Fe}_{70} \mathrm{Pd}_{30}$ material [45], whose properties have been summarized in Table 1.

\section{Temperature Induced Transformations}

SMAs evolve into complex microstructures below the transition temperature. In the following subsections, we study how the geometric aspect ratio and boundary conditions affect the microstructure morphology.
TABLE 1: $\mathrm{Fe}_{70} \mathrm{Pd}_{30}$ material constants.

\begin{tabular}{lc}
\hline$a_{1}$ & $192.3 \mathrm{GPa}$ \\
$a_{2}$ & $280 \mathrm{GPa}$ \\
$a_{3}$ & $19.7 \mathrm{GPa}$ \\
$a_{4}$ & $2.59 \times 10^{3} \mathrm{GPa}$ \\
$a_{5}$ & $8.52 \times 10^{4} \mathrm{Gpa}$ \\
$\eta$ & $0.25 \mathrm{~N}-\mathrm{s} \mathrm{m}^{2}$ \\
$k_{g}$ & $3.15 \times 10^{-8} \mathrm{~N}$ \\
$\theta_{m}$ & $270 \mathrm{~K}^{-1}$ \\
$\theta_{0}$ & $295 \mathrm{~K}^{-1}$ \\
$C_{v}$ & $350 \mathrm{Jkg}^{-1} \mathrm{~K}^{-1}$ \\
$\kappa$ & $78 \mathrm{~W} \mathrm{~m}^{-1} \mathrm{~K}^{-1}$ \\
$\rho$ & $10000 \mathrm{~kg} \mathrm{~m}^{-3}$ \\
\hline
\end{tabular}

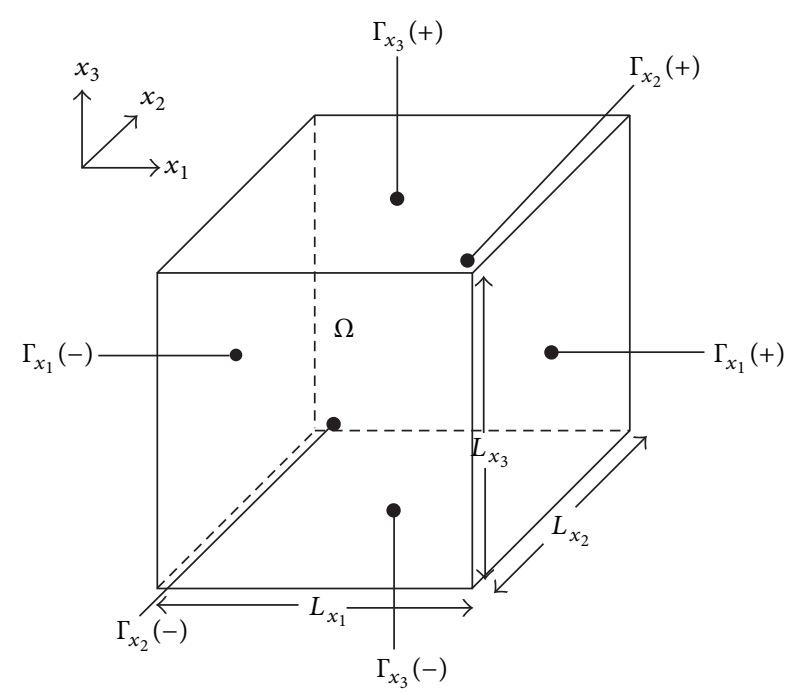

FIGURE 2: Schematic of the rectangular prism (domain $\Omega$ and nomenclature of boundaries $\Gamma$ ).

4.1. Influence of Geometric Aspect Ratio. Here, the simulations have been conducted on rectangular prism SMA specimens with $L_{x_{1}}=L_{x_{3}}=32 \mathrm{~nm}$ and three different thickness ratios $L_{x_{2}} / L_{x_{1}}=1 / 32,1 / 2,1$ representing a thinfilm, a slab, and a cube domain, respectively. The specimens are quenched to temperature corresponding to $\tau=-1.2$ and allowed to evolve until the energy and microstructures are stabilized. Fully periodic boundary conditions have been applied to the displacement vector $\mathbf{u}$ in all the simulations in Section 4.1. The stabilized martensitic microstructures at $t=$ $0.504 \mathrm{~ns}$ for these three domains are plotted in Figure 3. The red, blue, and green colors represent $M_{1}, M_{2}, M_{3}$ martensitic variants, respectively.

The microstructures in the SMA thin-film domain selfaccommodate to a twin morphology to minimize the energy as shown in Figure 3(a). Due to the thin-film geometry, the $\mathrm{M}_{2}$ variant is not allowed and not favored. The microstructure reveals only two variants $M_{1}$ and $M_{3}$ with a complete suppression of the out-of-plane variant $\mathrm{M}_{2}$. The microstructure is a sequence of $M_{1}$ and $M_{3}$ variant bands, which are evolved into approximately equal proportions as observed in Figure 4(a) 


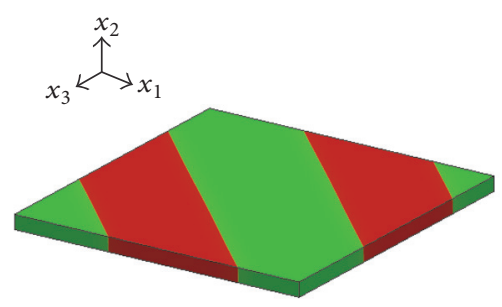

(a) Thin film

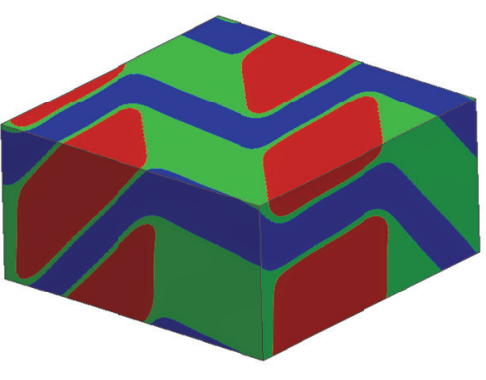

(b) Slab

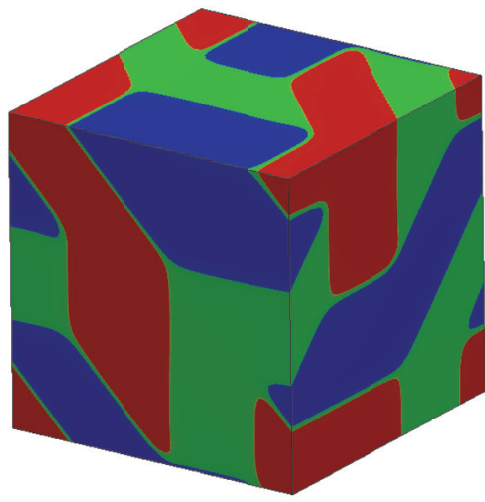

(c) Cube

Figure 3: (Color online) Self-accommodated microstructures in the (a) thin film, (b) slab, and (c) cube SMA domains (red, blue, and green colors represent $M_{1}, M_{2}$, and $M_{3}$ variants, resp.).

(refer to the dashed lines). The twin boundaries are aligned along the [101] plane. The stabilized microstructure reproduces the morphology previously reported in the 2D model $[49,54]$, the crystallographic theory [55], and experiments [56], thus verifying our numerical implementation and validating the results. The suppression of the out-of-plane variant in a thin-film has also been reported experimentally [57].

For energetic analysis, the energy density components $\left\langle\mathscr{F}_{\square}\right\rangle$ (in the dimensionless units) are plotted with time. Figure 4(b) reveals the $\left\langle\mathscr{F}_{\square}\right\rangle$ evolution for a thin-film specimen (refer to the solid lines). The autocatalytic nucleation starts at different locations in the domain at approximately $0.05 \mathrm{~ns}$, the domain gets deformed, leading to internal strain, which in turn transcends as the long-range elastic interactions causing the increase in $\left\langle\mathscr{F}_{g}\right\rangle$. Due to the strained state of the domain, the $\left\langle\mathscr{F}_{b}\right\rangle$ and $\left\langle\mathscr{F}_{s}\right\rangle$ increase. As the microstructure evolves, the $\langle\mathscr{F}\rangle$ is minimized with stabilization of energy components. The competition between $\left\langle\mathscr{F}_{g}\right\rangle,\left\langle\mathscr{F}_{b}\right\rangle$, and $\left\langle\mathscr{F}_{s}\right\rangle$ leads to the formation of the twinned microstructure.

The stabilized microstructure in the SMA slab domain is shown in Figure 3(b). The morphology reveals the primary bands of $M_{1}$ and $M_{3}$ variants, as observed in Figure 3(a); however, they are penetrated by the secondary band of the $\mathrm{M}_{2}$ variant. The three variants self-accommodate to form Chevron or herringbone patterns. The evolution to the approximately equal proportion of three martensitic variants is shown in Figure 4(a) (refer to the solid line). The observation of equal phase fractions of martensitic variants in the domains has been reported in the literature [58]. The width ratios of $M_{1}: M_{2}$ and $M_{3}: M_{2}$ bands are observed to be approximately $2: 1$. Similar width ratios have been observed numerically $[36,37]$ and experimentally in ferroelectric and ferroelastic ceramics $[59,60]$. The step-like morphology of $\mathrm{M}_{2}$ has also been reported experimentally [61]. The energetic analysis reveals that the nucleation and energy stabilization occur faster in the slab than in the thin-film domain. The $\left\langle\mathscr{F}_{g}\right\rangle$ dominates and is higher than the $\left\langle\mathscr{F}_{b}\right\rangle$ and $\left\langle\mathscr{F}_{s}\right\rangle$.

The microstructure morphology in the cube domain is distinct compared to the thin-film and slab domains as shown in Figure 3(c). The bands of $M_{1}$ and $M_{3}$ variants are not prominent as observed in Figures 3(a) and 3(b). Due to the periodic boundary conditions and elastic strain accommodation, the domain walls are aligned along the [110] planes and the microstructure forms polytwinned domains. Such accommodations of martensites have been reported in [36]. The three variants evolve in an equal phase fraction $\varphi$ as shown in Figure 4(a) (refer to the dash-dot lines).

Interestingly, the $\left\langle\mathscr{F}_{g}\right\rangle$ in the SMA cube specimen is smaller than in the slab specimen. The periodic boundary condition forces the slab domain to create more domain walls to maintain the equal proportion of martensitic variants in less volume (as compared to the cube domain) leading to a higher gradient energy.

The evolution of the average temperature coefficient $\tau$ for the three domains is plotted in Figure 4(c). A temperature increase is observed during microstructure evolution due to the thermomechanical coupling and insulated boundary conditions. The evolved temperature decreases with higher aspect ratios, due to the formation of fewer twins. This suggests that a better understanding of the thermomechanical coupling is especially important in $3 \mathrm{D}$, as different microstructure morphologies are evolved based on the aspect ratios that cannot be captured by the $2 \mathrm{D}$ models $[31,50]$.

Although all the above three simulations use periodic boundary conditions, it can be concluded that the aspect ratio of the domain plays an important role in the microstructure evolution. A lower aspect ratio promotes an equal proportion of martensitic variants, while a higher aspect ratio leads to suppression of the out-of-plane variant. Thus, by tuning the geometric aspect ratio, the domain patterns (microstructure) can be modified, which in turn can control preferential deformations for building special applications using SMAs [4].

4.2. Influence of Boundary Conditions. In this section, we study the influence of boundary conditions on the microstructure morphology of a $80 \mathrm{~nm}$ side SMA cube specimen. We start with random initial conditions in the 


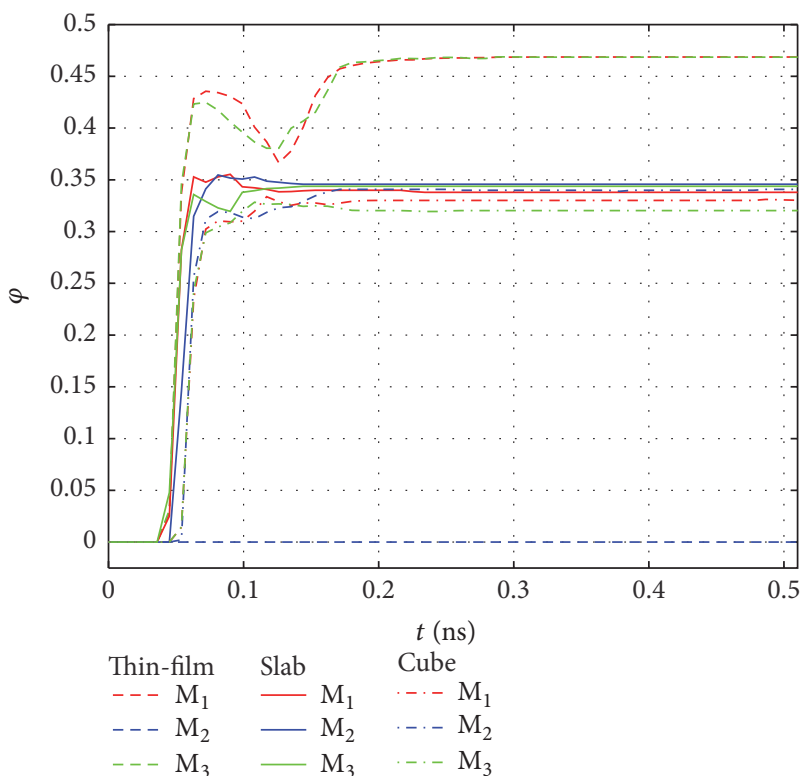

(a)

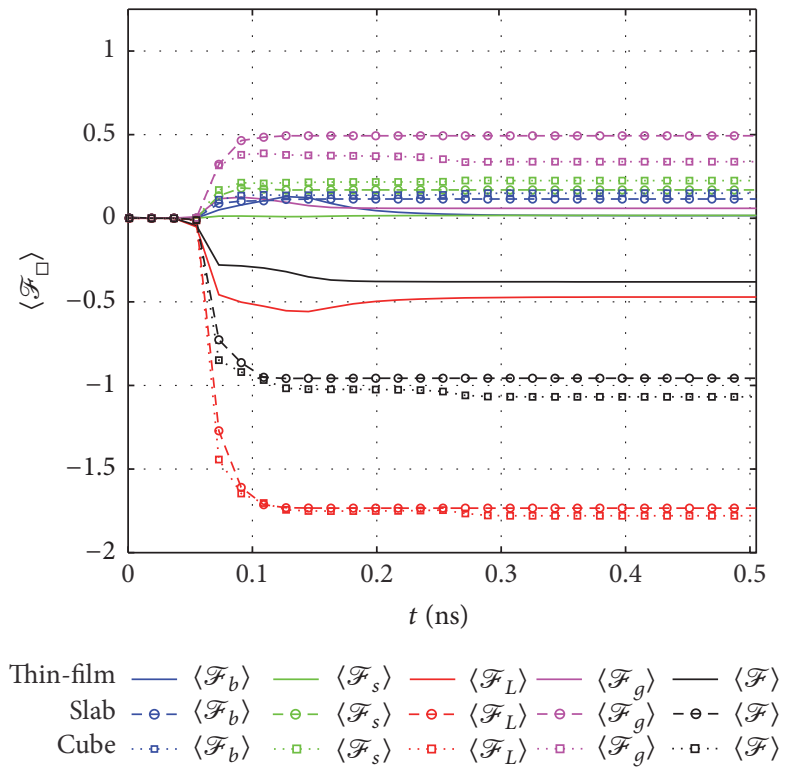

(b)

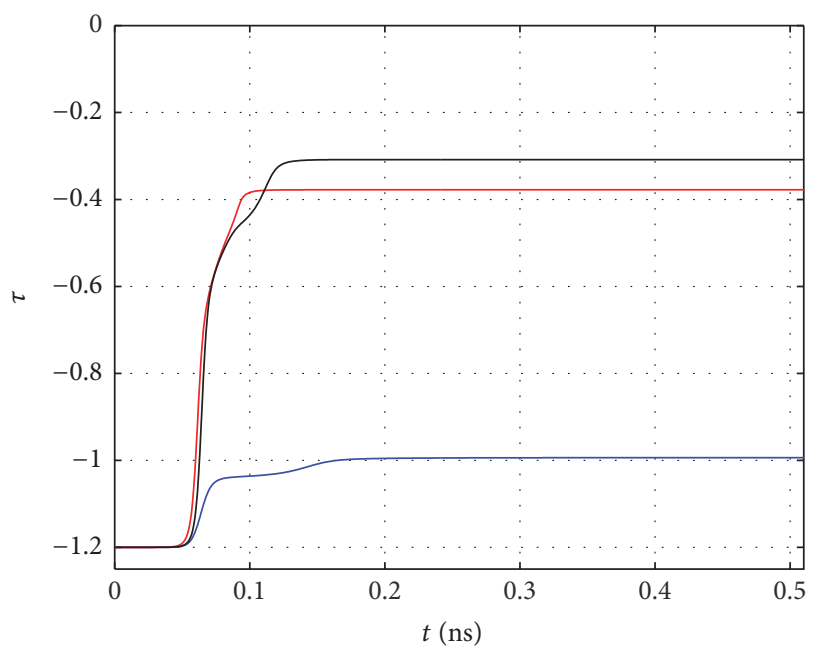

Thin-film
Slab
Cube

(c)

Figure 4: (Color online) Evolution of (a) phase fraction $\varphi$ (red, green, and blue colors refer to $\mathrm{M}_{1}, \mathrm{M}_{2}$, and $\mathrm{M}_{3}$ variants, resp.), (b) energy density, and (c) average temperature coefficient $\tau$ with time.

displacement vector $\mathbf{u}$. In all the simulations, the SMA specimen is quenched to temperature corresponding to $\tau=$ -1.2 and allowed to evolve for a sufficiently long time until the energy and microstructure are stabilized. Three different boundary conditions on $\Gamma$ have been used for the simulations: (i) fully constrained (FC) case with $\mathbf{u}=0$, (ii) normally constrained (NC) case with $\mathbf{u} \cdot \mathbf{n}=0$, and (iii) fully periodic (FP) case.

Figure 5 presents the stabilized microstructure morphology at $t=1.5 \mathrm{~ns}$ in the SMA cube domain with different boundary conditions. The sensitivity of the microstructure morphology evolution to the boundary conditions during temperature induced transformations is evident. The cube domain does not change the shape in the case of FC and $\mathrm{NC}$ boundary conditions. However, it can change the shape in the FP case.

Examining the FC boundary condition case in Figure 5(a) (a portion of the domain is not shown for viewing the variants inside the constrained surfaces), it is observed that the martensite variants are self-accommodated to form a complex microstructure morphology. The nucleation starts homogeneously, and the self-accommodation and stabilization of martensitic variants take place quickly into smaller lamella aligned along [110] planes. The mechanical constraints 


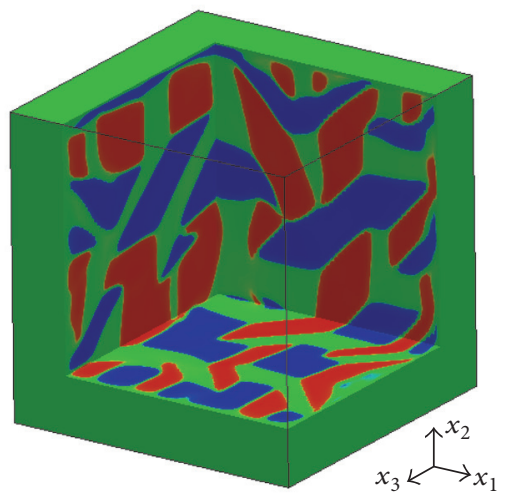

(a) Fully constrained

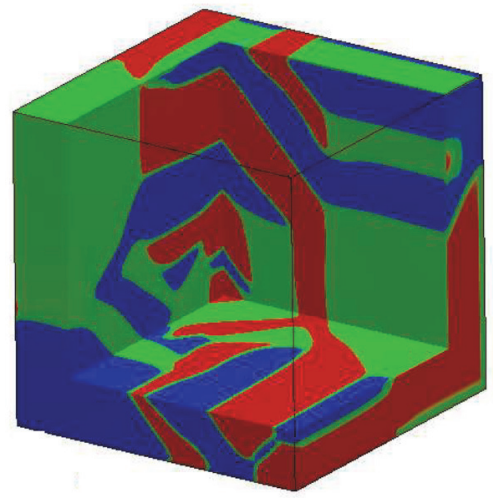

(b) Normally constrainted

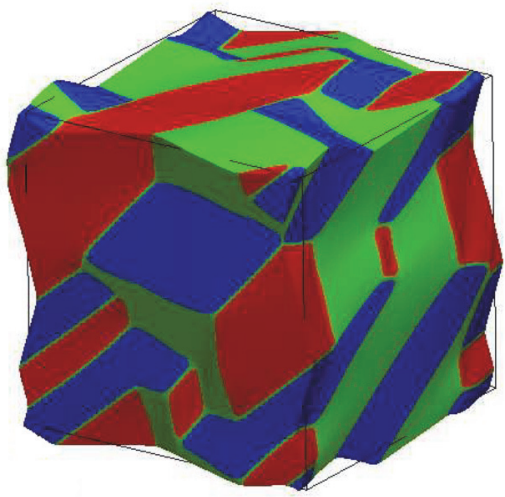

(c) Fully periodic

FIGURE 5: (Color online) Self-accommodated microstructure in a $80 \mathrm{~nm}$ side SMA cube under (a) fully constrained, (b) normally constrained, and (c) fully periodic boundary conditions (red, blue, and green colors represent $M_{1}, M_{2}$, and $M_{3}$ variants, resp.).

on surfaces $\Gamma$ forcefully introduce additional domain walls to maintain the size and shape of the cube domain. This leads to higher $\left\langle\mathscr{F}_{g}\right\rangle$ in the case of FC boundary conditions. The higher $\left\langle\mathscr{F}_{g}\right\rangle$ and additional domain walls lead to indistinguishable martensitic variant regions in the domain. Thus, the FC boundary condition case is governed by short-range effects. The $\left\langle\mathscr{F}_{s}\right\rangle$ dominates over the $\left\langle\mathscr{F}_{g}\right\rangle$ due to mechanical constraints. The $\langle\mathscr{F}\rangle$ is highest among all the cases. The martensitic variants evolve into equal proportions as shown in Figure 6(b).

The morphology for the NC boundary condition case is shown in Figure 5(b). Although the size and shape of the cube domain are constant, the martensitic variants are selfaccommodated in a plate morphology, in contrast with the FC boundary condition case. The nucleation starts homogeneously. The $\left\langle\mathscr{F}_{g}\right\rangle$ is smaller compared to the FC boundary condition case because the domain walls can move to selfaccommodate as the tangential displacement is allowed at the surface nodes. The bigger martensitic domains are a result of the long-range elastic interactions. Due to the system's ability for self-adjustment of the martensitic domain, the SMA specimen minimizes to a lower $\langle\mathscr{F}\rangle$ as compared to the FC boundary condition case.

The FP boundary condition morphology is shown in Figure 5(c). Due to the system's ability to change shape, the martensitic variants are self-accommodated in equal proportions. The $\left\langle\mathscr{F}_{g}\right\rangle$ dominates over the $\left\langle\mathscr{F}_{s}\right\rangle$. The nucleation is homogeneous and domain walls are aligned along [110] planes. Due to the flexibility of the system to change shape, the $\langle\mathscr{F}\rangle$ is lowest in the three boundary condition cases. The fully periodic system imposes an artificial constraint on the boundary to satisfy the continuity in degrees of freedom across the opposite surfaces of the cube.

The effect of different boundary conditions is also observed on the evolution of $\tau$, as shown in Figure 6(c). The boundary conditions and elastic interactions in the domain also affect the thermal behavior. The temperature gets stabilized quickly in the FC case, while it takes a comparatively long time for the FP boundary condition case.

\section{Stress Induced Transformations}

SMAs exhibit phase transformations and hysteretic properties during stress induced loadings. Here, the simulations have been conducted on SMA nanowires to understand the thermomechanical behavior under dynamic loading conditions. The current study focuses on the behavior of prismatic specimens. The study of microstructure morphology on complex 3D geometries like tube, tubular torus, and tubular spring using the developed model and isogeometric formulation have been reported in $[53,62]$. The shape-memory effect and pseudoelastic behaviors of SMA specimens have been studied in the following subsections.

5.1. Shape Memory Effect. SMAs reveal shape-memory effect (SME) hysteretic behavior below the transition temperature. A twinned martensite phase is transformed into the detwinned martensite phase under mechanical loading. The twinned microstructures are evolved in a rectangular prism nanowire of dimension $\left(L_{x_{1}} \times L_{x_{2}} \times L_{x_{3}}\right) 200 \times 40 \times 40 \mathrm{~nm}$. The simulation is conducted in two steps. First, the selfaccommodated martensitic variants are evolved in the SMA specimen starting from initial random conditions of the displacement vector $\mathbf{u}$. The specimen is quenched to the temperature corresponding to $\tau=-1.2$ and allowed to evolve until microstructure and energy are stabilized. The three martensitic variants evolve into approximately equal proportions as seen at time $t=0$ from the phase fraction $\varphi$ plot in Figure 8(c). On energy stabilization, the accommodated microstructure morphology is shown in Figure 7(a). During the microstructure evolution, a temperature increase is observed due to the thermomechanical coupling owing to the movements of domain walls and the adiabatic conditions. The evolved microstructure is taken as an initial condition to the tensile test in the second step.

The tensile test is conducted by constraining all displacements $(\mathbf{u}=0)$ on the surface $\Gamma_{x_{1}}(-)$ and axially loading and unloading the opposite surface $\Gamma_{x_{1}}(+)$ in the out-of-material plane direction using a ramp-based displacement loading and 


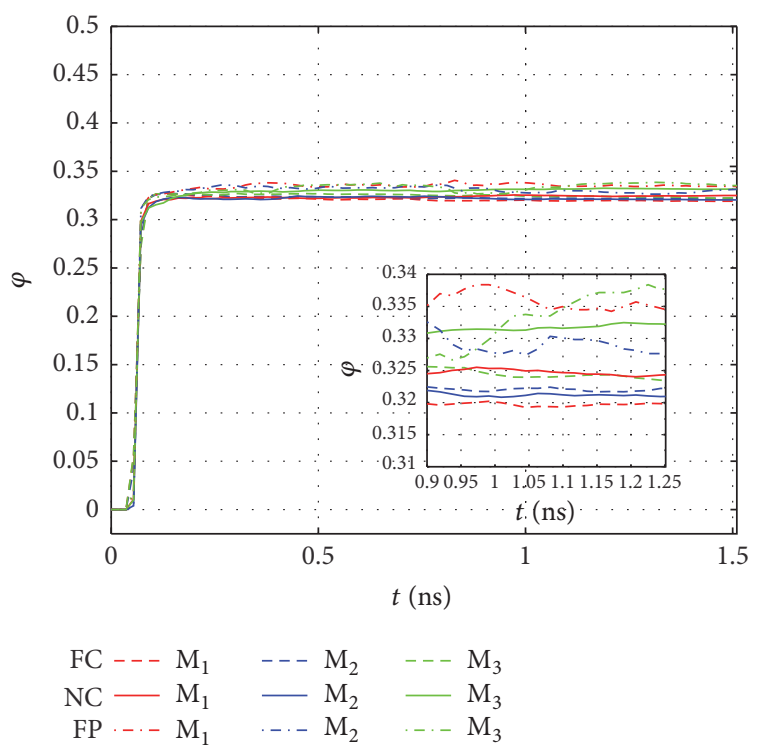

(a)

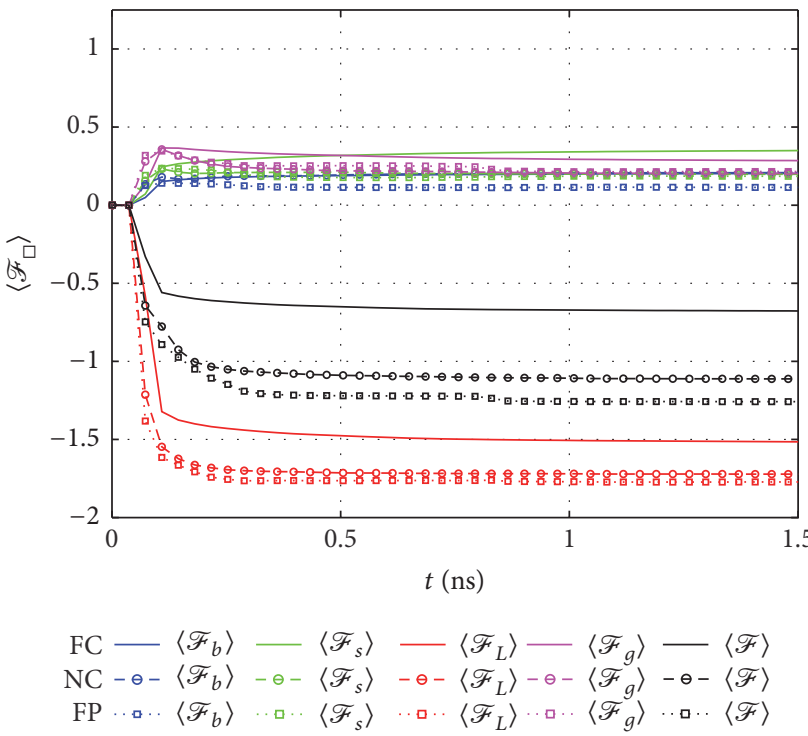

(b)

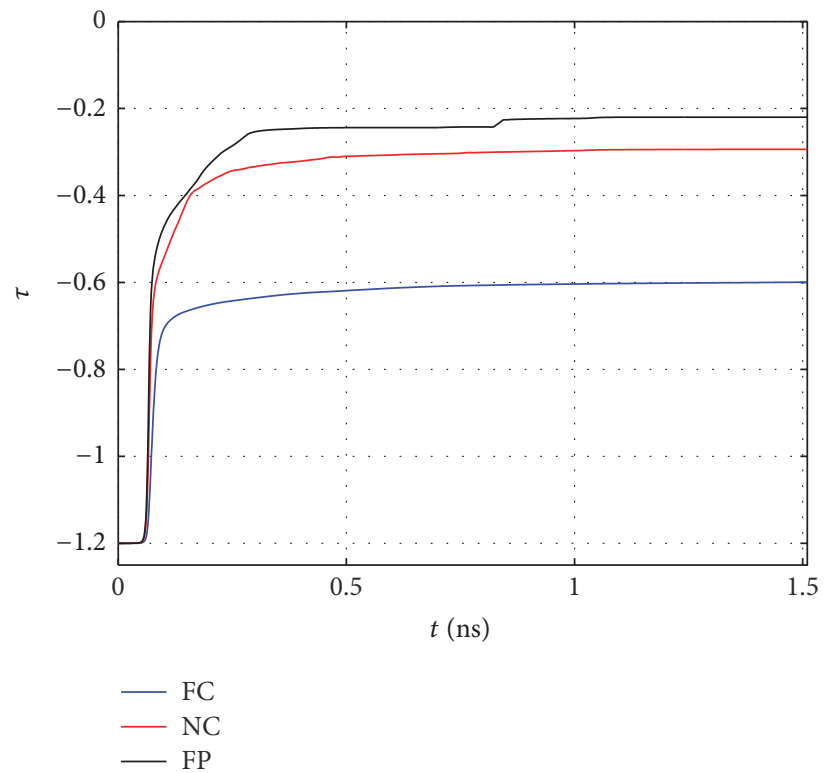

(c)

FiguRE 6: (Color online) Evolution of (a) phase fraction $\varphi$ (red, green, and blue colors refer to $\mathrm{M}_{1}, \mathrm{M}_{2}$, and $\mathrm{MM}_{3}$ variants, resp.), (b) energy density $\left\langle\mathscr{F}_{\square}\right\rangle$, and (c) average temperature coefficient $\tau$ with time. FC, NC, and FP represent fully constrained, normally constrained, and fully periodic boundary conditions, respectively.

unloading equivalent to the axial strain rate $\dot{\epsilon}_{11}$. The stressfree boundary conditions have been applied on the transverse surfaces $\left.\Gamma_{x_{i}}( \pm)\right|_{i=2,3}$.

The SME behavior of the nanowire is studied at axial strain rate $\dot{\epsilon}_{11}=14.4 \times 10^{7} / \mathrm{s}$ by achieving $3 \%$ axial strain in 0.208 ns. Figures $7(\mathrm{~b})-7(\mathrm{i})$ show time snapshots of the microstructure evolution during axial loading and unloading. The phase fraction evolution and thermomechanical behavior in SMA specimen are plotted in Figure 8. As the tensile test starts from a point $a$, the specimen is elastically loaded until it reaches point $b$. Because of the loading and boundary condition, phase transformations start at the ends of the nanowire. As the load progresses, the martensite variants start to coalesce to form distinct bands. The $\mathrm{M}_{2}$ and $\mathrm{M}_{3}$ variants start converting into the favorable $\mathrm{M}_{1}$ variant by the process of detwinning between points $b-h$. The motion, merging, and vanishing of $M_{2}$ and $M_{3}$ domains and growth of $M_{1}$ domain are apparent. During the loading, the domain walls move along the [110] planes. During the detwinning process, the axial stress is nearly constant between points $b-f$. The SMA specimen undergoes phase transformations $\left(\mathrm{M}_{2} \rightarrow\right.$ $M_{1}$ and $M_{3} \rightarrow M_{1}$ ) and elastic loading simultaneously between points $f-h$. After all the $\mathrm{M}_{2}$ and $\mathrm{M}_{3}$ are converted to the $M_{1}$ variant, the specimen is loaded elastically. During 


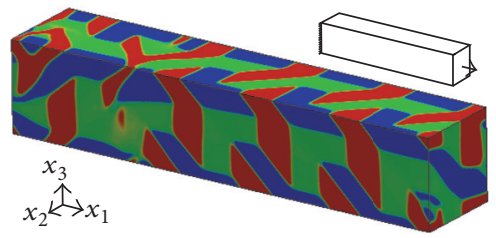

(a) $t=0 \mathrm{~ns}$

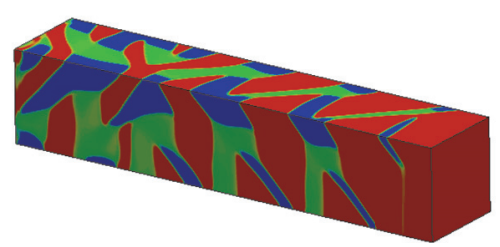

(d) $t=0.05 \mathrm{~ns}$

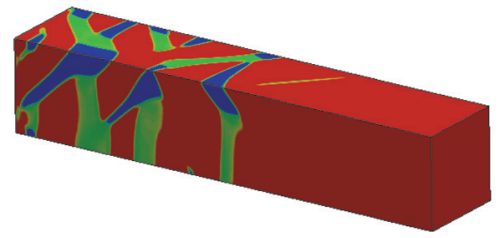

(g) $t=0.1 \mathrm{~ns}$

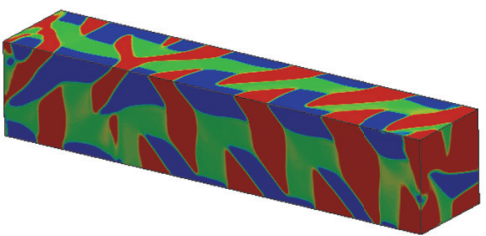

(b) $t=0.017 \mathrm{~ns}$

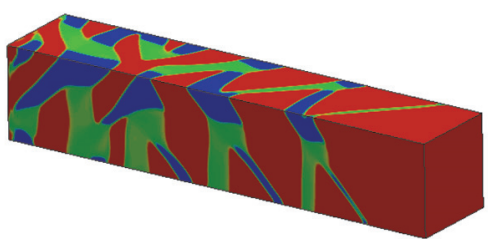

(e) $t=0.067 \mathrm{~ns}$

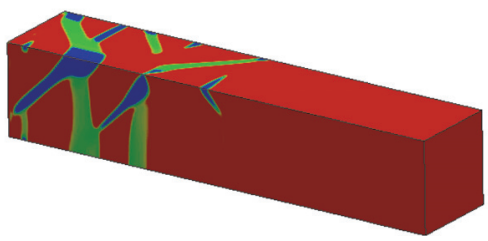

(h) $t=0.117 \mathrm{~ns}$

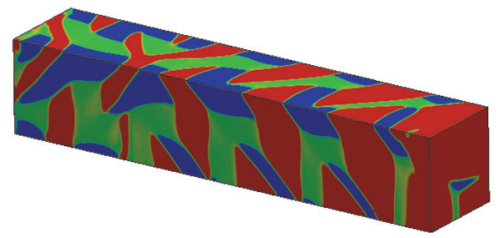

(c) $t=0.033 \mathrm{~ns}$

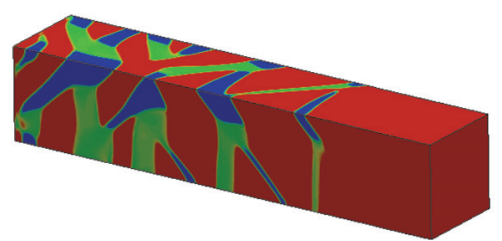

(f) $t=0.083 \mathrm{~ns}$

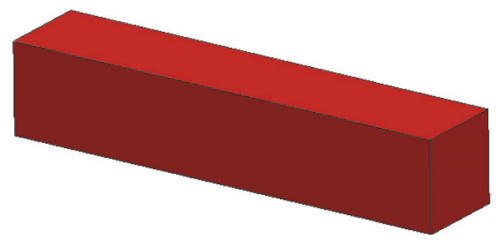

(i) $t=0.3 \mathrm{~ns}$

FIGURE 7: (Color online) SME: microstructure morphology evolution in a $200 \times 40 \times 40 \mathrm{~nm}$ nanowire (red, blue, and green colors represent $\mathrm{M}_{1}, \mathrm{M}_{2}$, and $\mathrm{M}_{3}$ variants, resp.).

unloading, the $M_{1}$ variant is elastically unloaded, till the stress in the domain is zero at the point $i$. The remnant axial strain is approximately $1.8 \%$ at the end of unloading. The effect of thermomechanical coupling is observed during the evolution of the average $\tau$ as shown in Figure 8(b). The increase and decrease of temperature are a result of exothermic and endothermic processes during loading and unloading of the nanowire. Similar behaviors of temperature increase and decrease have been observed experimentally during the dynamic loading-unloading of SMA specimens $[16,20,63,64]$.

As observed in Figures 7-8, the dynamic loading of SMAs involves growth, merging, and elimination of martensitic variants, domain wall movements, and temperature changes. It is now imperative to investigate how the domain size and strain rate affect these mechanisms and properties. In the following sections, the simulations focus on these aspects.

5.1.1. SME: Aspect Ratio Study. To investigate the influence of aspect ratios on the SME behavior, the simulations have been conducted on nanowires of four dimensions: (i) $200 \times 40 \times$ $40 \mathrm{~nm}$, (ii) $160 \times 40 \times 40 \mathrm{~nm}$, (iii) $80 \times 40 \times 40 \mathrm{~nm}$, and (iv) $160 \times 40 \times 80 \mathrm{~nm}$. All the simulations have been conducted according to the two-step procedure mentioned in the last section. The axial strain rate $\dot{\epsilon}_{11}=14.4 \times 10^{7} / \mathrm{s}$ is used.

Figure 9 presents the thermomechanical behavior on SMA nanowires of different aspect ratios. For the same lateral dimensions $\left(L_{x_{2}}=L_{x_{3}}=40 \mathrm{~nm}\right)$, the shorter length nanowire behaves in a stiffer manner and phase transformations occur at approximately constant axial stress.
This is because the deformation wave travels in a shorter domain, elastically loading the domain faster. The detwinning phase transformations, $\mathrm{M}_{2} \rightarrow \mathrm{M}_{1}$ and $\mathrm{M}_{3} \rightarrow \mathrm{M}_{1}$, occur at higher $\sigma_{11}$ value and have a distinct plateau.

The nanowires with lower $(160 \times 40 \times 40 \mathrm{~nm})$ and higher $(160 \times 40 \times 80 \mathrm{~nm})$ aspect ratios behave differently during loading. The detwinning phase transformation of a lower aspect ratio nanowires occurs at nearly constant $\sigma_{11}$, before the elastic loading of $\mathrm{M}_{1}$ after phase transformation is complete. In the case of high aspect ratio nanowires, the elastic loading and detwinning phase transformation occur simultaneously. The evolution of average $\tau$ for different aspect ratios shows similar trends, as presented in Figure 13(b).

5.1.2. SME: Strain Rate Study. The influence of strain rate on SME behavior is investigated on a SMA $160 \times 40 \times 40 \mathrm{~nm}$ nanowire. First, the SMA specimen is evolved to the selfaccommodated microstructure morphology by quenching it to the temperature coefficient $\tau=-1.2$ and allowing the energy and microstructures to stabilize. The specimen with evolved microstructures is then taken as an initial condition to the tensile test. The specimen is subjected to loading and unloading with different strain rates. The seven strain rates used during the studies are $9 \times 10^{7} / \mathrm{s}, 10.3 \times 10^{7} / \mathrm{s}, 12 \times 10^{7} / \mathrm{s}$, $14.4 \times 10^{7} / \mathrm{s}, 18 \times 10^{7} / \mathrm{s}, 24 \times 10^{7} / \mathrm{s}$, and $36 \times 10^{7} / \mathrm{s}$.

The thermomechanical behavior of the SMA nanowire at different strain rates is plotted in Figure 10. At lower strain rates between $9 \times 10^{7} / \mathrm{s}$ to $14.4 \times 10^{7} / \mathrm{s}$, a drop in $\sigma_{11}$ is observed during phase transformations. This is because during detwinning phase transformations $\left(M_{2} \rightarrow M_{1}\right.$ and 


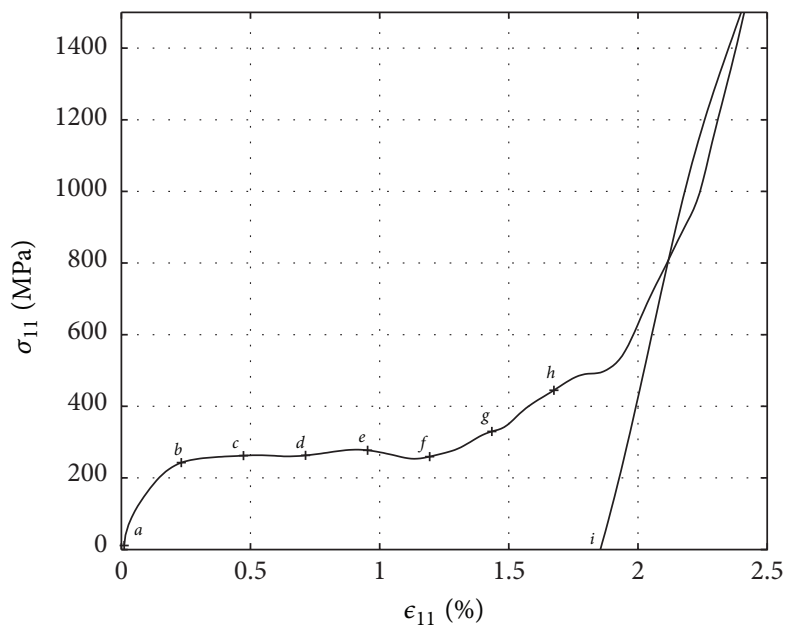

(a)

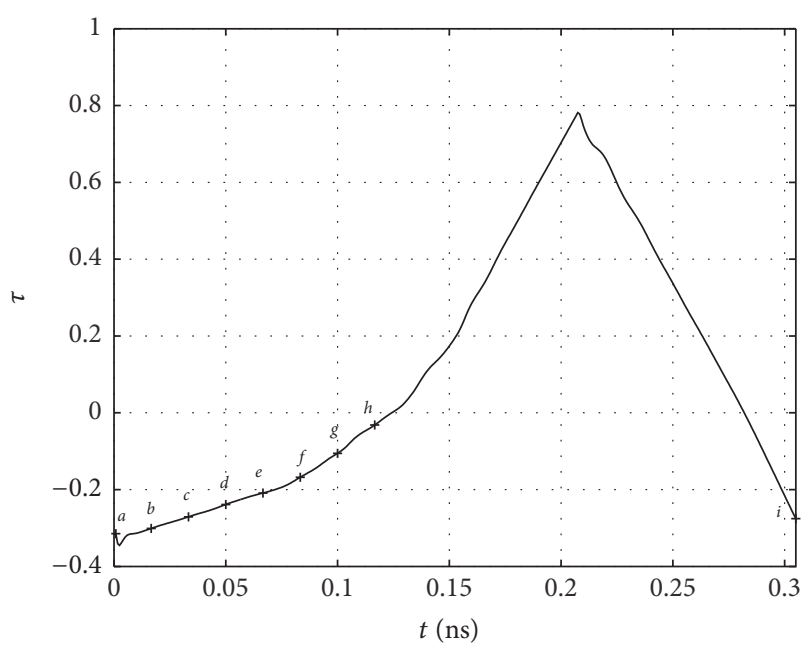

(b)

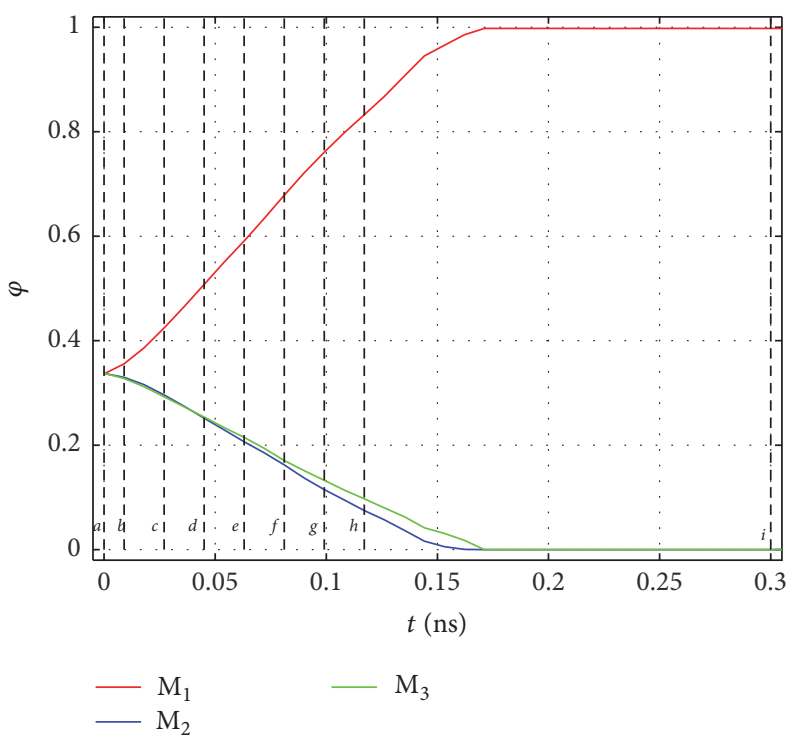

(c)

FIGURE 8: (Color online) SME: (a) the axial stress-strain $\left(\sigma_{11}-\epsilon_{11}\right)$ relation, and time evolution of (b) average $\tau$ and (c) phase fraction $\varphi$.

$\mathrm{M}_{3} \rightarrow \mathrm{M}_{1}$ ), the wire is relaxed axially for a short time before loading again. The phase transformation occurs and is completed at higher $\sigma_{11}$ values and at higher strain rates. At the intermediate strain rate of $18 \times 10^{7} / \mathrm{s}$, the phase transformation takes place at approximately constant $\sigma_{11}$. At higher strain rates like $24 \times 10^{7} / \mathrm{s}$ and $36 \times 10^{7} / \mathrm{s}$, the phase transformation and elastic loading take place simultaneously, and the phase transformation takes place at higher stress. As the SMA specimen does not have enough time to respond to the loading, the phase transformation no longer takes place at the constant stress value. The influence of strain rate is also evident on a temperature increase in a specimen as observed in Figure 10(b). The temperature increases faster in a nanowire at high strain rates. Similarly, the strain rate sensitivity on the thermomechanical behavior of SMA specimens has been reported during dynamic loading experiments $[20,63,64]$.
5.2. Pseudoelastic Behavior. SMAs exhibit pseudoelastic (PE) hysteretic behavior with complete recovery of strain above the transition temperature. We follow the two-step procedure mentioned in Section 5.1. The $200 \times 40 \times 40 \mathrm{~nm}$ SMA nanowire is evolved to the austenite phase with the temperature corresponding to $\tau=1.12$, starting with an initial random condition of displacement $\mathbf{u}$. The evolved austenite phase, as shown in Figure 11(a), is taken as an initial condition to the tensile test. In the following simulation, axial strain rate $\dot{\epsilon}_{11}=$ $3 \times 10^{7} / \mathrm{s}$ is used.

The time snapshots of microstructure morphology evolution, and thermomechanical properties of SMA specimen are presented in Figures 11(b)-11(i), and 12, respectively. Initially, the specimen is loaded elastically before the phase transformations start. The phase transformation $A \rightarrow M_{1}$ starts near the surface $\Gamma_{x_{1}}(+)$ as the loading progresses. The domain wall front moves towards the opposite end of the specimen 

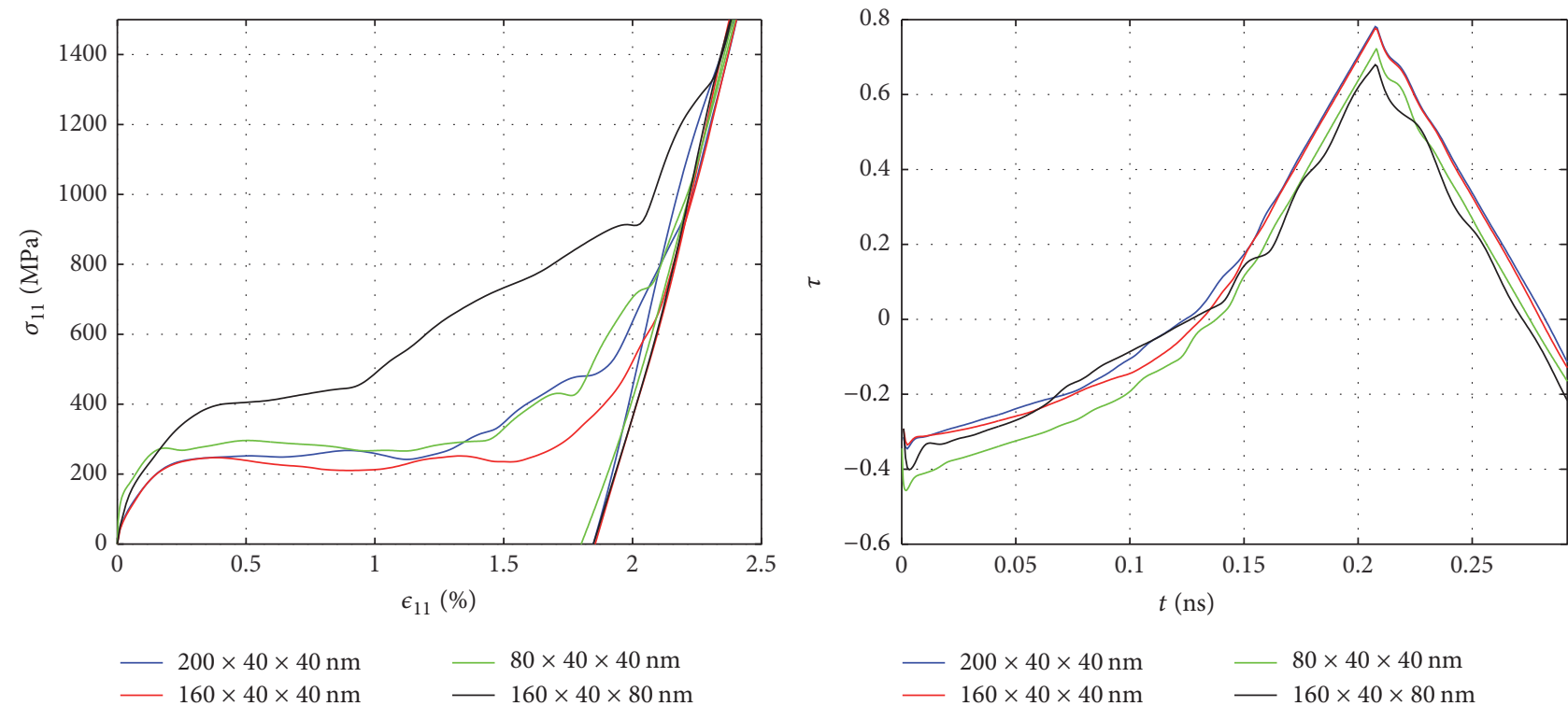

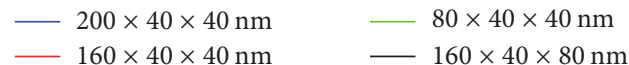

(a)

(b)

FIgURE 9: (Color online) SME aspect ratio study: (a) the average $\sigma_{11}-\epsilon_{11}$ behavior and (b) average evolution of $\tau$ with time.

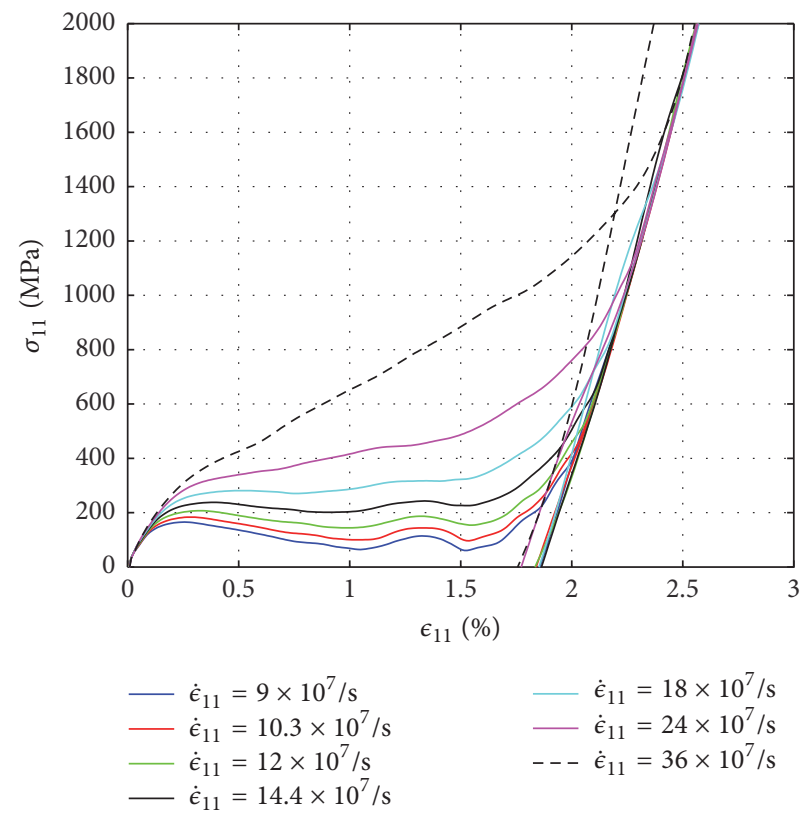

(a)

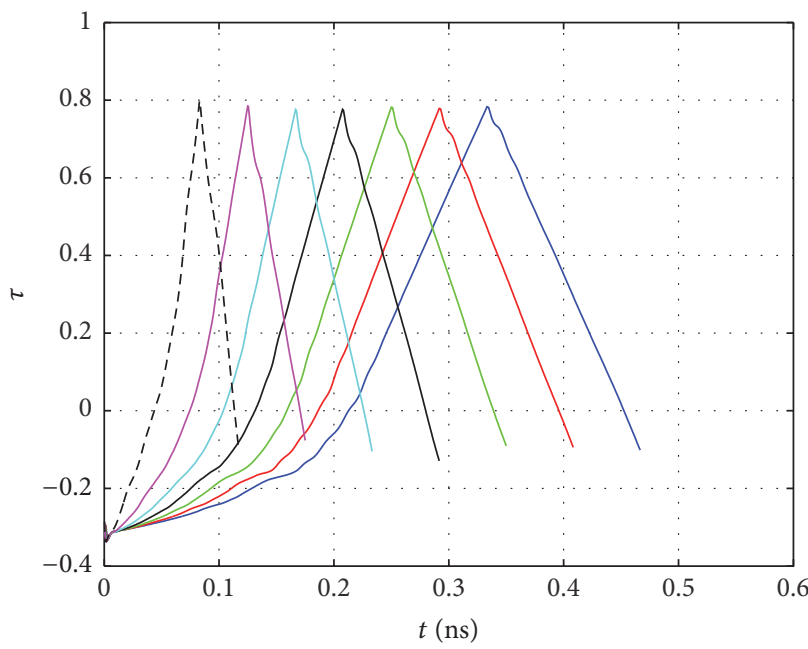

$\begin{aligned}-\dot{\epsilon}_{11} & =9 \times 10^{7} / \mathrm{s} \\ -\dot{\epsilon}_{11} & =10.3 \times 10^{7} / \mathrm{s} \\ -\dot{\epsilon}_{11} & =12 \times 10^{7} / \mathrm{s} \\ -\dot{\epsilon}_{11} & =14.4 \times 10^{7} / \mathrm{s}\end{aligned}$

$\dot{\epsilon}_{11}=18 \times 10^{7} / \mathrm{s}$

$-\dot{\epsilon}_{11}=24 \times 10^{7} / \mathrm{s}$

$---\dot{\epsilon}_{11}=36 \times 10^{7} / \mathrm{s}$

(b)

Figure 10: (Color online) SME strain rate study: (a) average $\sigma_{11}-\epsilon_{11}$ behavior and (b) average evolution of $\tau$ with time.

with the phase transformation. The phase transformation also nucleates near the $\Gamma_{x_{1}}(-)$ end. The combined phase transformation and axial loading occur at the later stages of loading, as observed with a steep rise in the axial $\sigma_{11}-\epsilon_{11}$ curve. The whole SMA specimen is converted into the $M_{1}$ phase towards the end of loading as shown in Figure 11(h). During the unloading, the reverse phase transformation $\mathrm{M}_{1} \rightarrow \mathrm{A}$ takes place with a complete recovery of strain at the end of unloading as shown in Figure 11(i). The influence of thermomechanical coupling on the average temperature coefficient $\tau$ is evident from Figure 12(b). The increase and decrease of $\tau$ are a result of exothermic and endothermic processes during loading and unloading of a specimen.

5.2.1. PE: Aspect Ratio Study. To investigate the influence of aspect ratios on a $\mathrm{PE}$ regime, the simulations have been conducted on nanowires of the same four dimensions as described in Section 5.1.1. The simulations have been 


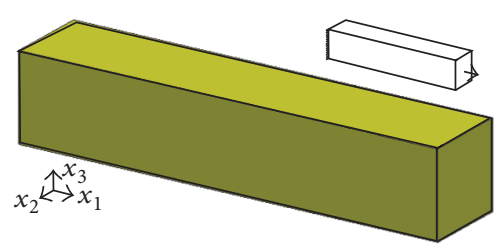

(a) $t=0 \mathrm{~ns}$

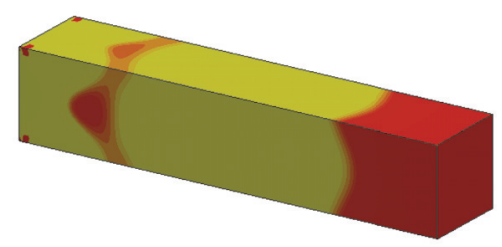

(d) $t=0.117 \mathrm{~ns}$

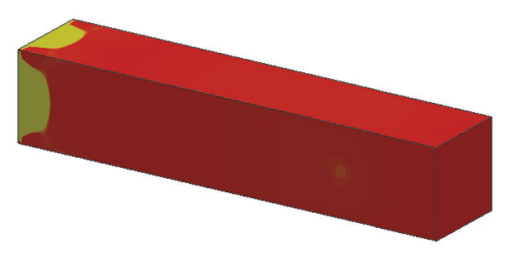

(g) $t=0.167 \mathrm{~ns}$

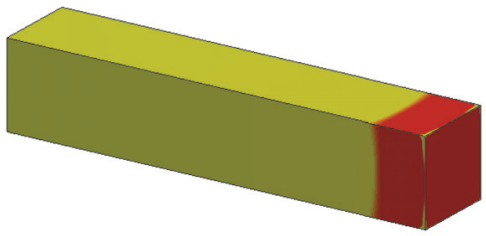

(b) $t=0.067 \mathrm{~ns}$

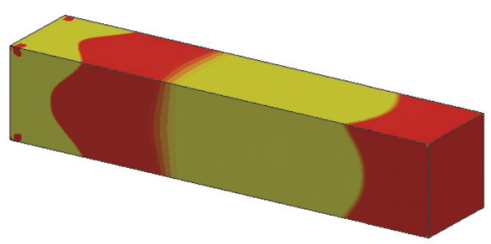

(e) $t=0.133 \mathrm{~ns}$

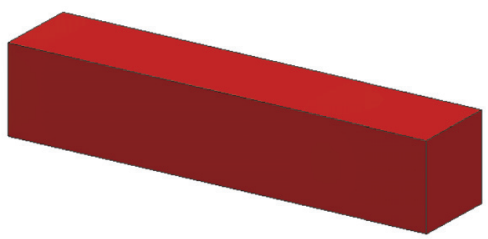

(h) $t=0.25 \mathrm{~ns}$

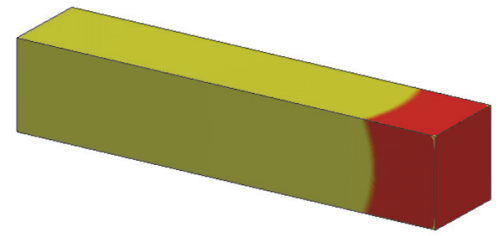

(c) $t=0.083 \mathrm{~ns}$

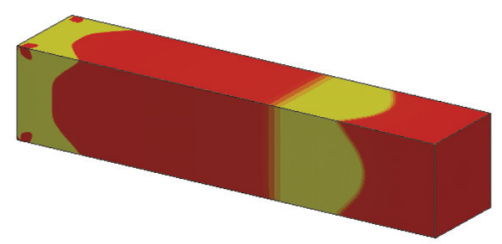

(f) $t=0.15 \mathrm{~ns}$

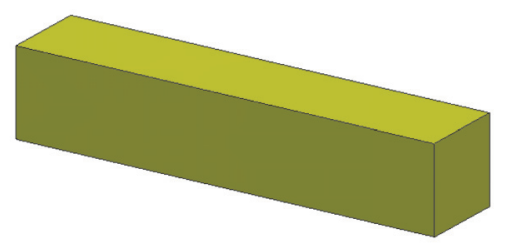

(i) $t=0.833 \mathrm{~ns}$

FIGURE 11: (Color online) PE: microstructure morphology evolution in a $200 \times 40 \times 40 \mathrm{~nm}$ nanowire (red and yellow colors represent $\mathrm{M}_{1}$ variant and austenite phase).

conducted following the two-step procedure mentioned in the previous section, but now evolving the domains at the temperature corresponding to $\tau=1.12$, starting with an initial random condition of displacement $\mathbf{u}$. The axial strain rate $\dot{\epsilon}_{11}$ $=3 \times 10^{7} / \mathrm{s}$ is used.

Figure 13 illustrates the thermomechanical behavior of nanowires with different aspect ratios. For the same lateral dimensions $\left(L_{x_{2}}=L_{x_{3}}=40 \mathrm{~nm}\right)$, the shorter length nanowire behaves in a stiffer manner during elastic loading. For a particular axial strain, the elastic loading of longer nanowires takes place at lower $\sigma_{11}$. Thus, the shorter nanowire behaves in a stiffer manner, as the loading deformation wave travels quicker. The phase transformation $\left(A \rightarrow M_{1}\right)$ occurs at nearly constant $\sigma_{11}$. During unloading, the phase transformation $\left(\mathrm{M}_{1} \rightarrow \mathrm{A}\right)$ completes early in shorter nanowires. Eventually, the nanowire returns to its original shape, without remnant strain in the system.

The nanowires with lower $(160 \times 40 \times 40 \mathrm{~nm})$ and higher $(160 \times 40 \times 80 \mathrm{~nm})$ aspect ratios behave similarly during loading. However, during unloading, the phase transformation is completed early in high-aspect ratio nanowires. The evolution of average $\tau$ for different aspect ratios shows similar trends, as presented in Figure 13(b).

5.2.2. PE: Strain Rate Study. The strain rate influence on PE behavior is studied on the $160 \times 40 \times 40 \mathrm{~nm}$ SMA nanowire. The specimen is subjected to loading and unloading at three different strain rates: $3 \times 10^{7} / \mathrm{s}, 4 \times 10^{7} / \mathrm{s}$, and $6 \times 10^{7} / \mathrm{s}$.

The thermomechanical behavior of SMA nanowires at different strain rates is plotted in Figure 14. It is apparent from
Figure 14(a) that the elastic loading and phase transformation $\left(\mathrm{A} \rightarrow \mathrm{M}_{1}\right)$ processes are distinct at lower strain rates. However, at higher strain rates, the elastic loading and phase transformation occur simultaneously. The competition between loading rates and the evolution dynamics plays an important role. The influence of mechanical loading is observed in a specimen with temperature increase and decrease during loading, as seen from Figure 14(b).

\section{Conclusions}

(i) A new fully coupled dynamic thermomechanical 3D phase-field model has been developed for cubicto-tetragonal phase transformations in SMAs. The model has a bidirectional coupling via temperature, strain, and strain rate. The governing equations have been numerically implemented in a variational form based on the isogeometric analysis, which allows for straightforward implementation of the fourth order differential terms using the rich NURBS basis functions.

(ii) The aspect ratio of the domain plays an important role in the microstructure morphology evolution during thermally induced transformations. The lower aspect ratio promotes the equal proportion of martensitic variants, while the higher aspect ratio leads to suppression of the out-of-plane variant. The gradient energy in a slab is higher than in the cube specimen in order to maintain an equal phase fraction of the martensitic variants in the domain. 


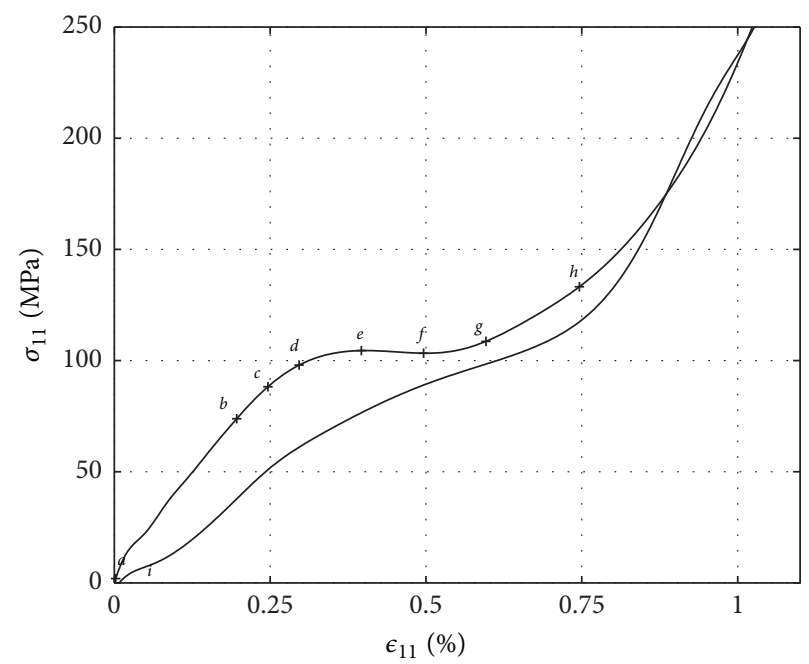

(a)

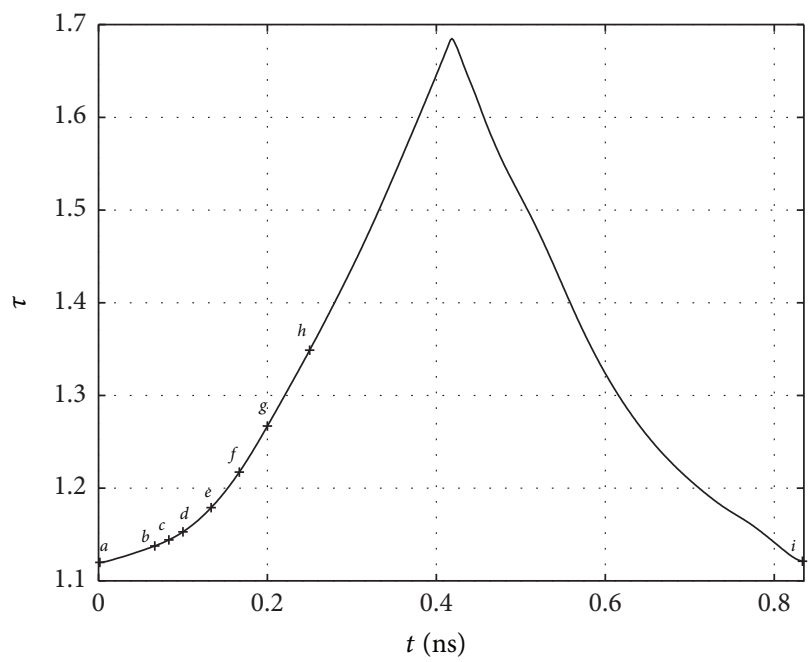

(b)

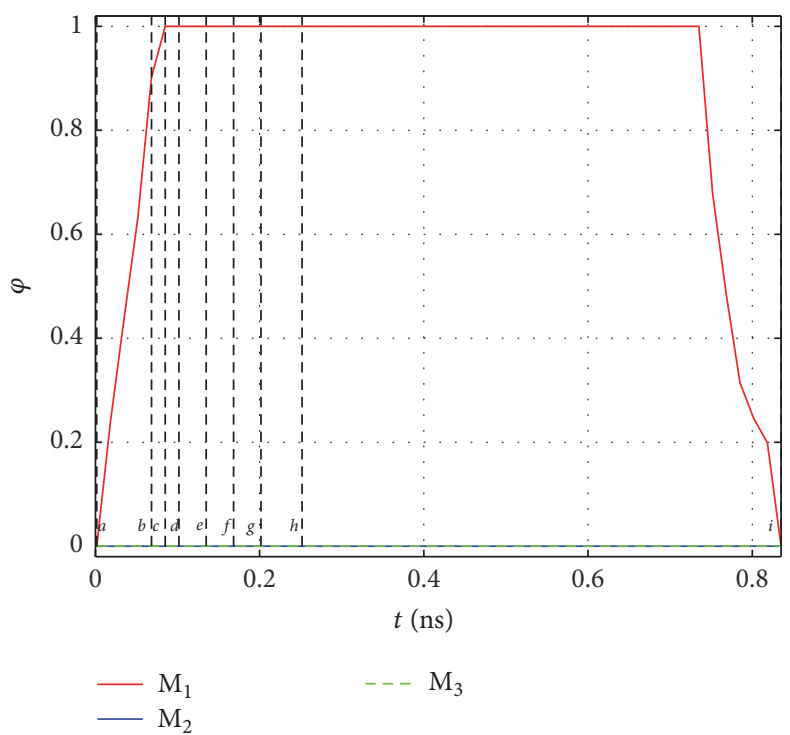

(c)

FIgURE 12: (Color online) PE: (a) axial stress-strain $\left(\sigma_{11}-\epsilon_{11}\right)$ relation, and time evolution of (b) average $\tau$ and (c) phase fraction $\varphi$.

(iii) During thermally induced transformations, the boundary conditions have a sensitive influence on the microstructure morphology. The SMA specimen with fully constrained boundary conditions is stabilized in the shortest time, followed by normally constrained and then fully periodic boundary conditions. Energetically, the fully constrained boundary condition has the highest free energy, and the fully periodic condition has the lowest. The fully periodic boundary condition allows the formation of distinct selfaccommodated domains due to long-range elastic interactions.

(iv) The model reproduces the shape memory effect and pseudoelastic behavior in the dynamic tensile loading-unloading test. The influence of mechanical loading on temperature evolution is in agreement with the experiments reported in the literature.

(v) The tensile test on rectangular prisms of different aspect ratios reveals the impact of geometry on the thermomechanical behavior of SMAs. For the same lateral dimensions, the shorter length nanowire behaves in a stiffer manner and phase transformations occur at approximately constant axial stress. In the higher aspect ratio specimen, during SME loading, the elastic loading and phase transformations occur simultaneously. In the case of PE, the loading behavior is similar; however the unloading phase transformation is completed earlier.

(vi) The lower strain rate loading causes a phase transformation to take place at constant axial stress, with 


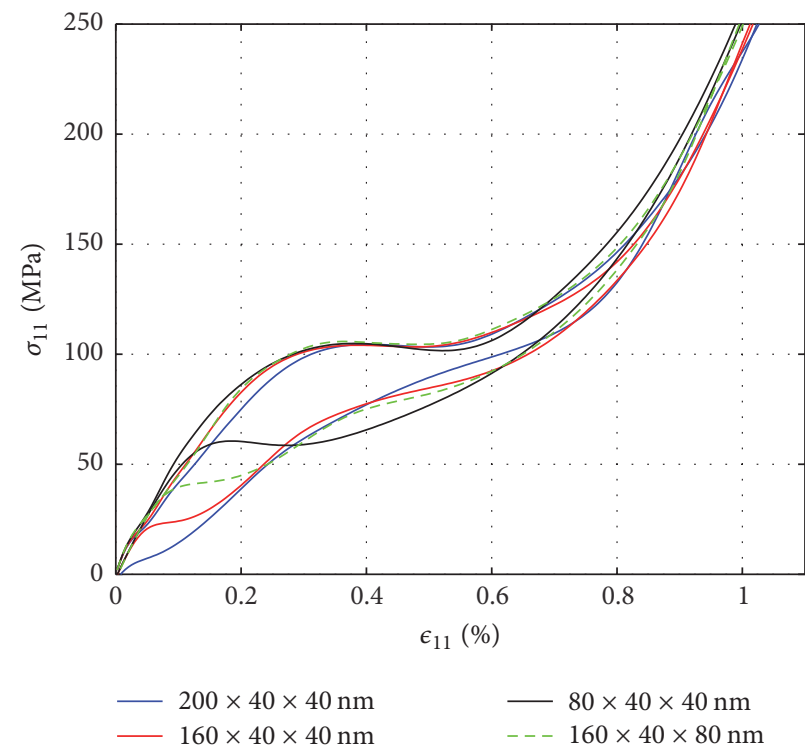

(a)

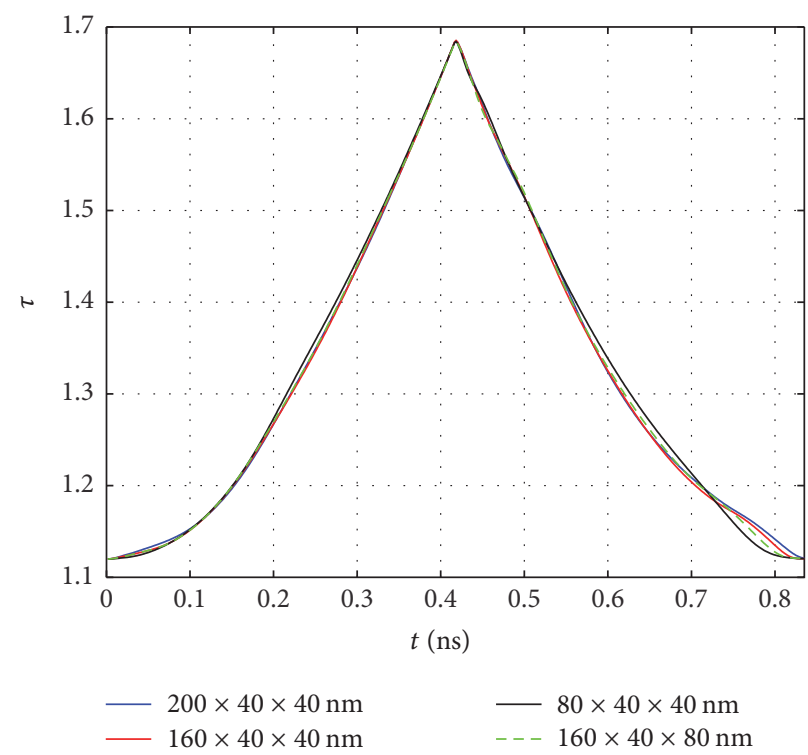

(b)

FIgURE 13: (Color online) PE aspect ratio study: (a) average $\sigma_{11}-\epsilon_{11}$ behavior and (b) average evolution of $\tau$ with time.

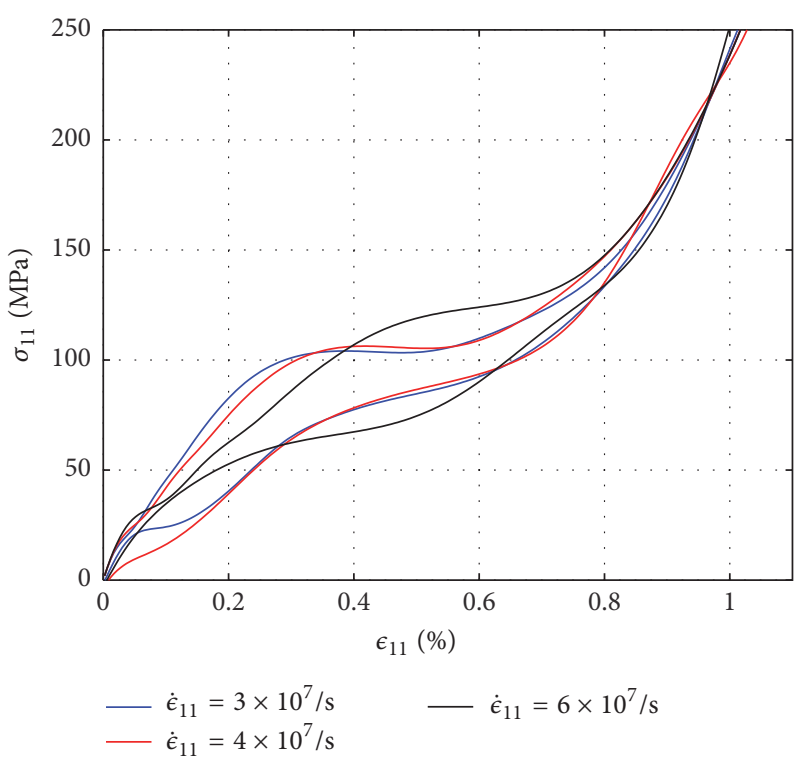

(a)

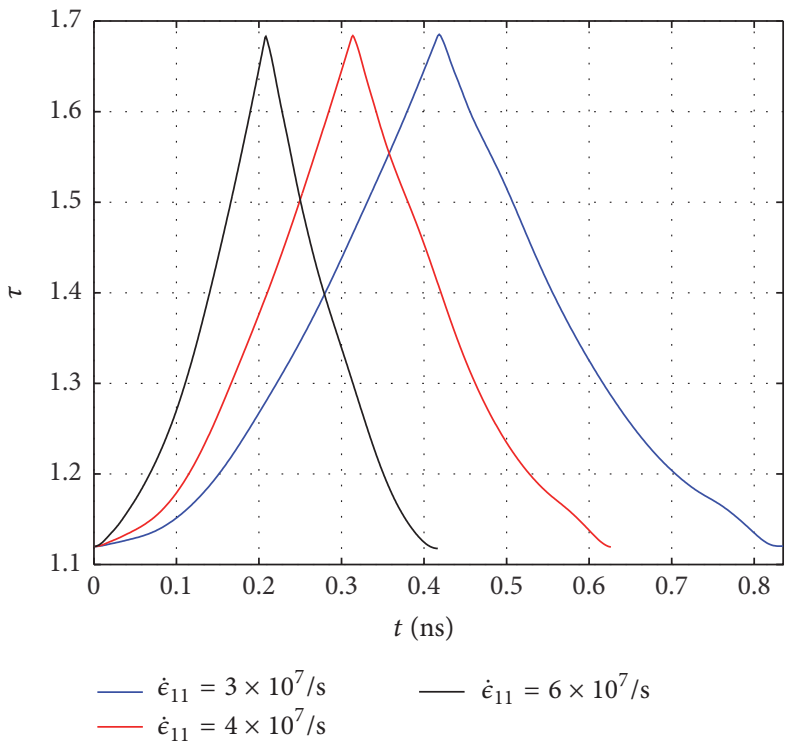

(b)

FIGURE 14: (Color online) PE strain rate study: (a) average $\sigma_{11}-\epsilon_{11}$ behavior and (b) average evolution of $\tau$ with time.

a distinct plateau. However, at higher strain rates, the elastic loading and phase transformation occur simultaneously. The dynamics of phase transformations and loading rate, as well as the time scale of twin interface motion, play an important role.

Our model and numerical framework is a step forward in better understanding the dynamics of thermomechanical behavior of SMA nanostructures for the development of novel SMA-based applications.
In the future, the generalized Ginzburg-Landau free energy [65] that accounts for various phase transformations along with the free energy due to orientation degree of freedom [66] can be used to model polycrystalline ceramic materials. There is a possibility to extend this study to understand the effect of dislocations on SMA properties by incorporating dislocation theory with a free-energy functional written in terms of the symmetry-adapted components of the elastic strain tensor [67]. 


\section{Appendix}

\section{Stress Components}

The stress tensor components $\left\{\sigma_{i j}\right\}$ are

$$
\begin{aligned}
\sigma_{11} & =\frac{a_{1} e_{1}}{\sqrt{3}}+\frac{e_{2}}{\sqrt{2}}\left[2 \tau a_{3}-6 a_{4} e_{3}+4 a_{5}\left(e_{2}^{2}+e_{3}^{2}\right)\right] \\
& +\frac{1}{\sqrt{6}}\left[e_{3}\left(2 \tau a_{3}+4 a_{5}\left(e_{2}^{2}+e_{3}^{2}\right)\right)+3 a_{4}\left(e_{3}^{2}-e_{2}^{2}\right)\right] \\
\sigma_{12} & =\sigma_{21}=\frac{1}{2} a_{2} e_{6} \\
\sigma_{13} & =\sigma_{31}=\frac{1}{2} a_{2} e_{5} \\
\sigma_{22} & =\frac{a_{1} e_{1}}{\sqrt{3}}-\frac{e_{2}}{\sqrt{2}}\left[2 \tau a_{3}-6 a_{4} e_{3}+4 a_{5}\left(e_{2}^{2}+e_{3}^{2}\right)\right] \\
& +\frac{1}{\sqrt{6}}\left[e_{3}\left(2 \tau a_{3}+4 a_{5}\left(e_{2}^{2}+e_{3}^{2}\right)\right)+3 a_{4}\left(e_{3}^{2}-e_{2}^{2}\right)\right] \\
\sigma_{23} & =\sigma_{32}=\frac{1}{2} a_{2} e_{4}, \\
\sigma_{33} & =\frac{1}{\sqrt{3}} a_{1} e_{1} \\
& -\frac{2}{\sqrt{6}}\left[2 \tau a_{3} e_{3}+3 a_{4}\left(e_{3}^{2}-e_{2}^{2}\right)+4 a_{5} e_{3}\left(e_{2}^{2}+e_{3}^{2}\right)\right]
\end{aligned}
$$

\section{Competing Interests}

The authors declare that there is no conflict of interests regarding the publication of this paper.

\section{Acknowledgments}

R. Dhote, R. Melnik, and J. Zu have been supported by the NSERC and CRC program (R. Melnik), Canada. R. Melnik thanks colleagues at Mevlana University for their hospitality during his visit there and TÜBITAK for its support. H. Gomez was partially supported by the European Research Council through the FP7 Ideas Starting Grant program (Project \#307201) and by Consellería de Educación e Ordenación Universitaria (Xunta de Galicia). Their support is gratefully acknowledged. This work was made possible with the facilities of the Shared Hierarchical Academic Research Computing Network (SHARCNET: https://www.sharcnet.ca) and Compute/Calcul Canada.

\section{References}

[1] D. Lagoudas, Shape Memory Alloys: Modeling and Engineering Applications, Springer, London, UK, 2008.

[2] K. Otsuka and C. Wayman, Shape Memory Materials, Cambridge University Press, 1998.

[3] K. Žužek Rožman, D. Pečko, S. Šturm et al., "Electrochemical synthesis and characterization of $\mathrm{Fe}_{70} \mathrm{Pd}_{30}$ nanotubes for drugdelivery applications," Materials Chemistry and Physics, vol. 133, no. 1, pp. 218-224, 2012.
[4] K. Bhattacharya and R. D. James, "The material is the machine," Science, vol. 307, no. 5706, pp. 53-54, 2005.

[5] D. Konig, M. Ehmann, S. Thienhaus, and A. Ludwig, "Microto nanostructured devices for the characterization of scaling effects in shape-memory thin films," Journal of Microelectromechanical Systems, vol. 19, no. 5, pp. 1264-1269, 2010.

[6] B. C. Bayer, S. Sanjabi, C. Baehtz et al., "Carbon nanotube forest growth on NiTi shape memory alloy thin films for thermal actuation," Thin Solid Films, vol. 519, no. 18, pp. 6126-6129, 2011.

[7] K. Clements, "Wireless technique for microactivation," US Patent 6,588,208, 2003.

[8] J. M. San Juan, M. L. Nó, and C. A. Schuh, "Superelasticity and shape memory in micro- and nanometer-scale pillars," Advanced Materials, vol. 20, no. 2, pp. 272-278, 2008.

[9] J. S. Juan, M. L. Nó, and C. A. Schuh, "Nanoscale shapememory alloys for ultrahigh mechanical damping," Nature Nanotechnology, vol. 4, no. 7, pp. 415-419, 2009.

[10] W. L. Benard, H. Kahn, A. H. Heuer, and M. A. Huff, "Thinfilm shape-memory alloy actuated micropumps," Journal of Microelectromechanical Systems, vol. 7, no. 2, pp. 245-251, 1998.

[11] M. Kohl, Shape Memory Microactuators, Springer, Berlin, Germany, 2004.

[12] S. Miyazaki, Y. Q. Fu, and W. M. Huang, Thin Film Shape Memory Alloys: Fundamentals and Device Applications, Cambridge University Press, Cambridge, UK, 2009.

[13] T. Yoneyama and S. Miyazaki, Shape Memory Alloys for Biomedical Applications, CRC Press, 2009.

[14] D. Zakharov, G. Lebedev, A. Irzhak et al., "Submicron-sized actuators based on enhanced shape memory composite material fabricated by FIB-CVD," Smart Materials and Structures, vol. 21, no. 5, Article ID 052001, 2012.

[15] F. R. Phillips, D. Fang, H. Zheng, and D. C. Lagoudas, "Phase transformation in free-standing SMA nanowires," Acta Materialia, vol. 59, no. 5, pp. 1871-1880, 2011.

[16] J. A. Shaw and S. Kyriakides, "Thermomechanical aspects of NiTi," Journal of the Mechanics and Physics of Solids, vol. 43, no. 8, pp. 1243-1281, 1995.

[17] P. H. Leo, T. W. Shield, and O. P. Bruno, "Transient heat transfer effects on the pseudoelastic behavior of shape-memory wires," Acta Metallurgica et Materialia, vol. 41, no. 8, pp. 2477-2485, 1993.

[18] J. A. Shaw and S. Kyriakides, "On the nucleation and propagation of phase transformation fronts in a NiTi alloy," Acta Materialia, vol. 45, no. 2, pp. 683-700, 1997.

[19] S. P. Gadaj, W. K. Nowacki, and E. A. Pieczyska, “Temperature evolution in deformed shape memory alloy," Infrared Physics \& Technology, vol. 43, no. 3-5, pp. 151-155, 2002.

[20] E. A. Pieczyska, S. P. Gadaj, W. K. Nowacki, and H. Tobushi, "Thermomechanical investigations of martensitic and reverse transformations in TiNi shape memory alloy," Bulletin of the Polish Academy of Sciences: Technical Sciences, vol. 52, no. 3, pp. 165-171, 2004.

[21] E. A. Pieczyska, S. P. Gadaj, W. K. Nowacki, and H. Tobushi, "Phase-transformation fronts evolution for stress- and straincontrolled tension tests in tini shape memory alloy," Experimental Mechanics, vol. 46, no. 4, pp. 531-542, 2006.

[22] E. Pieczyska and H. Tobushi, "Temperature evolution in shape memory alloy during loading in various conditions," in Proceedings of the 10th International Conference on Quantitative InfraRed Thermography, pp. 1-6, Quebec, Canada, June 2010. 
[23] Y. J. He and Q. P. Sun, "Rate-dependent domain spacing in a stretched NiTi strip," International Journal of Solids and Structures, vol. 47, no. 20, pp. 2775-2783, 2010.

[24] F. Falk, "Model free energy, mechanics, and thermodynamics of shape memory alloys," Acta Metallurgica, vol. 28, no. 12, pp. 1773-1780, 1980.

[25] F. Falk, "One-dimensional model of shape memory alloys," Archives of Mechanics, vol. 35, no. 1, pp. 63-84, 1983.

[26] G. Olson and M. Cohen, Dislocation Theory of Martensitic Transformations, vol. 7, North-Holland, 1986.

[27] A. Khandelwal and V. Buravalla, "Models for shape memory alloy behavior: an overview of modeling approaches," The International Journal of Structural Changes in Solids, vol. 1, no. 1, pp. 111-148, 2011.

[28] M. Mamivand, M. A. Zaeem, and H. El Kadiri, "A review on phase field modeling of martensitic phase transformation," Computational Materials Science, vol. 77, pp. 304-311, 2013.

[29] L.-Q. Chen, "Phase-field models for microstructure evolution," Annual Review of Materials Science, vol. 32, pp. 113-140, 2002.

[30] V. I. Levitas and D. L. Preston, "Three-dimensional landau theory for multivariant stress-induced martensitic phase transformations. I. Austenite $\leftrightarrow$ martensite," Physical Review BCondensed Matter and Materials Physics, vol. 66, no. 13, Article ID 134206, pp. 1342061-1342069, 2002.

[31] M. Bouville and R. Ahluwalia, "Microstructure and mechanical properties of constrained shape-memory alloy nanograins and nanowires," Acta Materialia, vol. 56, no. 14, pp. 3558-3567, 2008.

[32] E. Salje, Phase Transitions in Ferroelastic and Co-Elastic Crystals, Cambridge Topics I, Cambridge University Press, Cambridge, UK, 1993.

[33] N. Provatas and K. Elder, Phase-Field Methods in Materials Science and Engineering, Wiley-VCH, 2010.

[34] G. R. Barsch and J. A. Krumhansl, "Twin boundaries in ferroelastic media without interface dislocations," Physical Review Letters, vol. 53, no. 11, pp. 1069-1072, 1984.

[35] G. Barsch and J. Krumhansl, Nonlinear Physics in Martensitic Transformations in Martensite, ASM International, Materials Park, Ohio, USA, 1992.

[36] A. E. Jacobs, S. H. Curnoe, and R. C. Desai, "Simulations of cubic-tetragonal ferroelastics," Physical Review B-Condensed Matter and Materials Physics, vol. 68, no. 22, pp. 22410412241047, 2003.

[37] Y. Wang and A. G. Khachaturyan, "Three-dimensional field model and computer modeling of martensitic transformations," Acta Materialia, vol. 45, no. 2, pp. 759-773, 1997.

[38] A. Artemev, Y. Jin, and A. G. Khachaturyan, "Threedimensional phase field model of proper martensitic transformation," Acta Materialia, vol. 49, no. 7, pp. 1165$1177,2001$.

[39] V. I. Levitas and D. L. Preston, “Three-dimensional landau theory for multivariant stress-induced martensitic phase transformations. II. Multivariant phase transformations and stress space analysis," Physical Review B, vol. 66, no. 13, Article ID 134207, 15 pages, 2002.

[40] V. I. Levitas, D. L. Preston, and D.-W. Lee, “Three-dimensional Landau theory for multivariant stress-induced martensitic phase transformations. III. Alternative potentials, critical nuclei, kink solutions, and dislocation theory," Physical Review B, vol. 68, no. 13, Article ID 134201, 24 pages, 2003.

[41] A. V. Idesman, J.-Y. Cho, and V. I. Levitas, "Finite element modeling of dynamics of martensitic phase transitions," Applied Physics Letters, vol. 93, no. 4, Article ID 043102, 2008.
[42] V. I. Levitas and M. Javanbakht, "Surface tension and energy in multivariant martensitic transformations: phase-field theory, simulations, and model of coherent interface," Physical Review Letters, vol. 105, no. 16, Article ID 165701, 2010.

[43] V. I. Levitas and M. Javanbakht, "Surface-induced phase transformations: multiple scale and mechanics effects and morphological transitions," Physical Review Letters, vol. 107, no. 17, Article ID 175701, 2011.

[44] R. Ahluwalia, T. Lookman, A. Saxena, and S. R. Shenoy, "Pattern formation in ferroelastic transitions," Phase Transitions, vol. 77, no. 5-7, pp. 457-467, 2004.

[45] R. Ahluwalia, T. Lookman, and A. Saxena, "Dynamic strain loading of cubic to tetragonal martensites," Acta Materialia, vol. 54, no. 8, pp. 2109-2120, 2006.

[46] D. J. Seol, S. Y. Hu, Y. L. Li, J. Shen, K. H. Oh, and L. Q. Chen, "Computer simulation of spinodal decomposition in constrained films," Acta Materialia, vol. 51, no. 17, pp. 5173-5185, 2003.

[47] J. Man, J. Zhang, Y. Rong, and N. Zhou, "Study of thermoelastic martensitic transformations using a phase-field model," Metallurgical and Materials Transactions A, vol. 42, no. 5, pp. 11541164, 2011.

[48] Y. Ni, Y. M. Jin, and A. G. Khachaturyan, "The transformation sequences in the cubic $\rightarrow$ tetragonal decomposition," Acta Materialia, vol. 55, no. 14, pp. 4903-4914, 2007.

[49] R. P. Dhote, R. V. N. Melnik, and J. Zu, "Dynamic thermomechanical coupling and size effects in finite shape memory alloy nanostructures," Computational Materials Science, vol. 63, pp. 105-117, 2012.

[50] R. P. Dhote, H. Gomez, R. N. V. Melnik, and J. Zu, "Isogeometric analysis of a dynamic thermo-mechanical phase-field model applied to shape memory alloys," Computational Mechanics, vol. 53, no. 6, pp. 1235-1250, 2014.

[51] R. V. N. Melnik, A. J. Roberts, and K. A. Thomas, "Phase transitions in shape memory alloys with hyperbolic heat conduction and differential-algebraic models," Computational Mechanics, vol. 29, no. 1, pp. 16-26, 2002.

[52] J. Cottrell, T. Hughes, and Y. Bazilevs, Isogeometric Analysis: Toward Integration of CAD and FEA, John Wiley \& Sons, 2009.

[53] R. P. Dhote, H. Gomez, R. N. V. Melnik, and J. Zu, "3D coupled thermo-mechanical phase-field modeling of shape memory alloy dynamics via isogeometric analysis," Computers \& Structures, vol. 154, pp. 48-58, 2015.

[54] R. Dhote, H. Gomez, R. Melnik, and J. Zu, "Phase field dynamic modelling of shape memory alloys based on isogeometric analysis," Advances in Science and Technology, vol. 78, pp. 6368, 2013.

[55] J. Sapriel, “Domain-wall orientations in ferroelastics," Physical Review B, vol. 12, no. 11, pp. 5128-5140, 1975.

[56] K. Bhattacharya, Microstructure of Martensite: Why It Forms and How It Gives Rise to the Shape-Memory Effect, Oxford University Press, 2003.

[57] Y. Ma, A. Setzer, J. W. Gerlach, F. Frost, P. Esquinazi, and S. G. Mayr, "Freestanding single crystalline Fe-Pd ferromagnetic shape memory membranes-role of mechanical and magnetic constraints across the martensite transition," Advanced Functional Materials, vol. 22, no. 12, pp. 2529-2534, 2012.

[58] Y. Cui, J. Wan, J. Zhang, and Y. Rong, "Strain-induced phase transition in martensitic alloys: phase-field simulation," in Proceedings of the PRICM: 8 Pacific Rim International Congress on Advanced Materials and Processing, pp. 2781-2790, Wiley Online Library, Waikoloa, Hawaii, USA, 2013. 
[59] G. Arlt, "Twinning in ferroelectric and ferroelastic ceramics: stress relief," Journal of Materials Science, vol. 25, no. 6, pp. 26552666, 1990.

[60] N. A. Pertsev and G. Arlt, "Theory of the banded domain structure in coarse-grained ferroelectric ceramics," Ferroelectrics, vol. 132, no. 1, pp. 27-40, 1992.

[61] P. Boullay, D. Schryvers, and J. M. Ball, "Nano-structures at martensite macrotwin interfaces in Ni65Al35," Acta Materialia, vol. 51, no. 5, pp. 1421-1436, 2003.

[62] R. P. Dhote, H. Gomez, R. N. Melnik, and J. Zu, "Shape memory alloy nanostructures with coupled dynamic thermo-mechanical effects," Computer Physics Communications, vol. 192, pp. 48-53, 2015.

[63] S. P. Gadaj, W. K. Nowacki, and E. A. Pieczyska, "Temperature evolution in deformed shape memory alloy," Infrared Physics and Technology, vol. 43, no. 3-5, pp. 151-155, 2002.

[64] E. Pieczyska and H. Tobushi, "Temperature evolution in shape memory alloy during loading in various conditions," in Proceedings of the 10th International Conference on Quantitative InfraRed Thermography, pp. 5-10, Québec, Canada, July 2010.

[65] R. Ahluwalia, S. Vedantam, T. Lookman, and A. Saxena, A Generalized Ginzburg-Landau Model for Martensitic Transformations in Shape Memory Alloys, Minerals, Metals and Materials Society/AIME, Warrendale, Pa, USA, 2011.

[66] R. Ahluwalia, T. Lookman, A. Saxena, and R. C. Albers, "Landau theory for shape memory polycrystals," Acta Materialia, vol. 52, no. 1, pp. 209-218, 2004.

[67] R. Gröger, B. Marchand, and T. Lookman, "Dislocations via incompatibilities in phase-field models of microstructure evolution," Physical Review B, vol. 94, no. 5, Article ID 054105, 2016. 


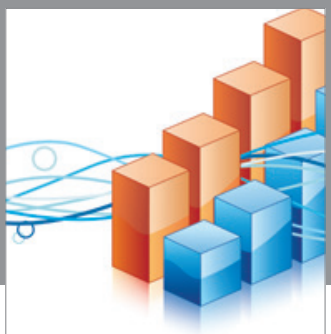

Advances in

Operations Research

vatem alat4

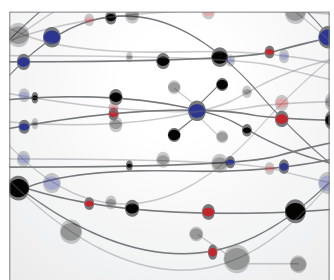

\section{The Scientific} World Journal
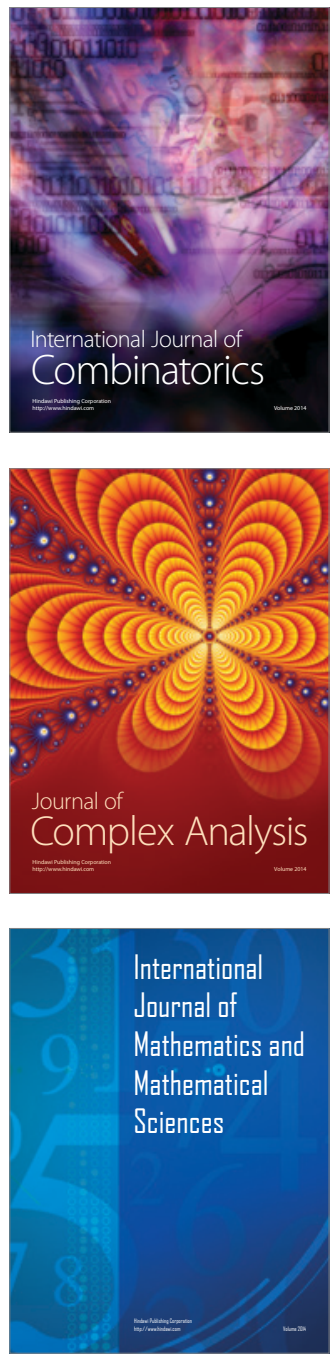
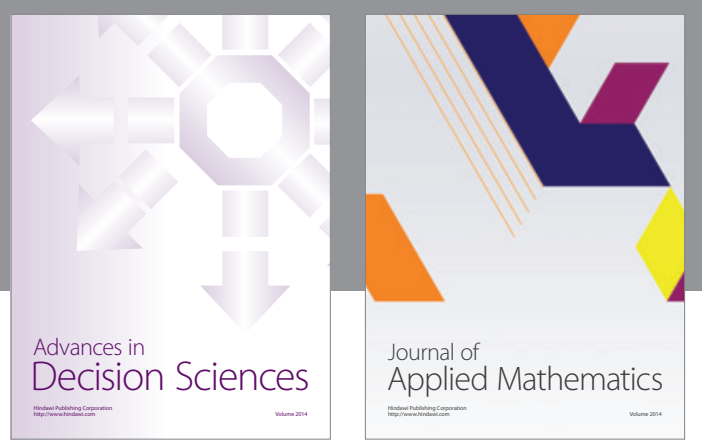

Algebra

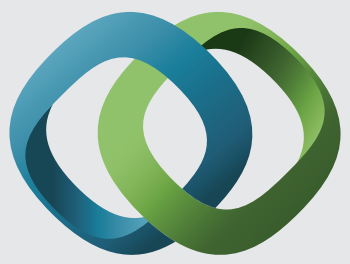

\section{Hindawi}

Submit your manuscripts at

http://www.hindawi.com
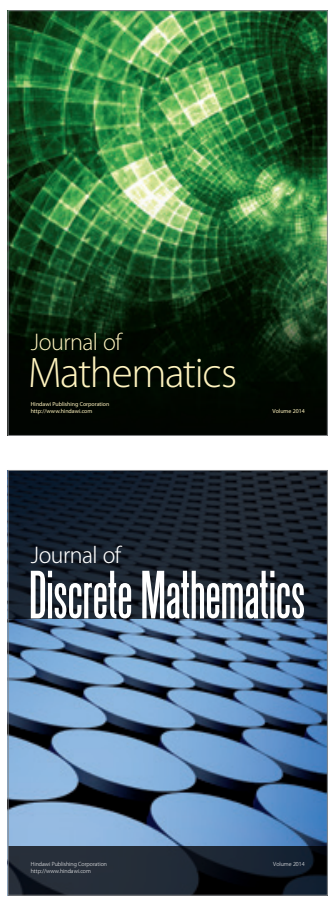

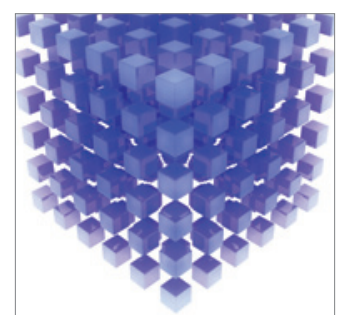

Mathematical Problems in Engineering
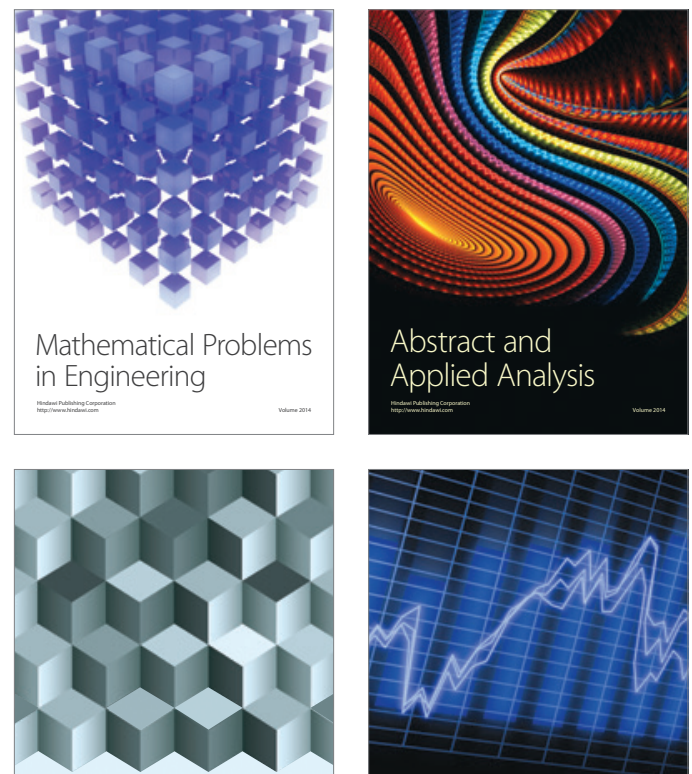

Journal of

Function Spaces

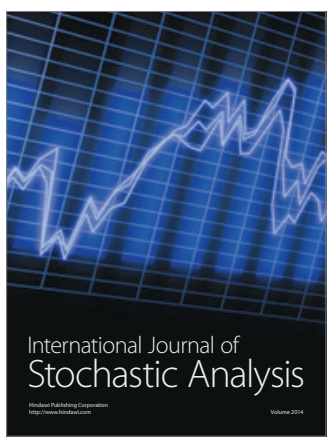

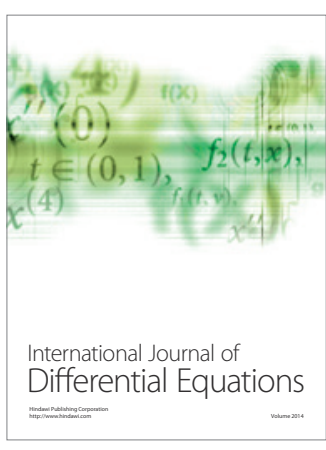
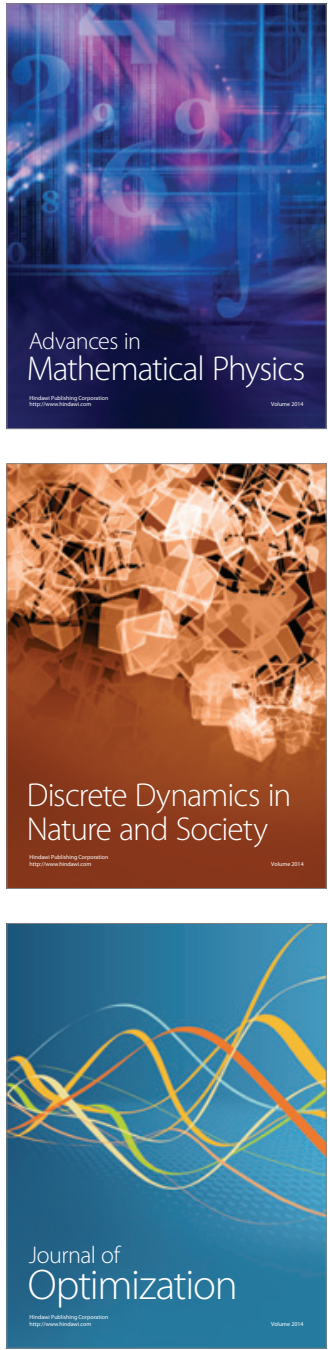